\title{
Going against the norm: validation of a novel alternative to brain SPECT normative datasets
}

\author{
Lindsay M. Quandt ${ }^{*}{ }^{*}$, Cyrus A. Raji² \\ ${ }^{1}$ CereHealth Corporation, Littleton, CO 80120, USA \\ ${ }^{2}$ Mallinckrodt Institute of Radiology, Washington University in St. Louis, St. Louis, MO 63110, USA
}

*Correspondence: Lindsay M. Quandt, CereHealth Corporation, Littleton, C0 80120, USA. LQuandt@ceremetrix.io Academic Editor: Rhoda Au, Boston University School of Medicine, USA

Received: April 23, 2020 Accepted: September 20, 2020 Published: October 30, 2020

Cite this article: Quandt LM, Raji CA. Going against the norm: validation of a novel alternative to brain SPECT normative datasets. Explor Med. 2020;1:331-54. https://doi.org/10.37349/emed.2020.00022

\begin{abstract}
Aim: Quantitative analysis of brain single photon emission computed tomography (SPECT) perfusion imaging is dependent on normative datasets that are challenging to produce. This study investigated the combination of SPECT neuroimaging from a large clinical population rather than small numbers of controls. The authors hypothesized this "population template" would demonstrate noninferiority to a control dataset, providing a viable alternative for quantifying perfusion abnormalities in SPECT neuroimaging.

Methods: A total of 2, 068 clinical SPECT scans were averaged to form the "population template". Validation was three-fold. First, the template was imported into SPECT brain analysis software, MIMneuro ${ }^{\circledR}$, and compared against its control dataset of 90 individuals through its region and cluster analysis tools. Second, a cohort of 100 cognitively impaired subjects was evaluated against both the population template and MIMneuro ${ }^{\circledR} \mathrm{S}$ normative dataset to compute region-based metrics. Concordance and intraclass correlation coefficients, mean square deviations, total deviation indices, and limits of agreement were derived from these data to measure agreement and test for noninferiority. Finally, the same patients were clinically read in CereMetrix ${ }^{\circledR}$ to confirm that expected perfusion patterns appeared after comparison to the template.

Results: MIMneuro ${ }^{\circledR \prime}$ 's default threshold for normality is $\pm 1.65 \mathrm{z}$-score and this served as our noninferiority margin. Direct comparison of the template to controls produced no regions that exceeded this threshold and all clusters identified were far from statistically significant. Agreement measures revealed consistency between the softwares and that CereMetrix ${ }^{\circledR}$ results were noninferior to MIMneuro ${ }^{\circledR}$, albeit with proportional bias. Visual analysis also confirmed that expected perfusion patterns appeared when individual scans were compared to the population template within CereMetrix ${ }^{\circledR}$.

Conclusions: The authors demonstrated a population template was noninferior to a smaller control dataset despite inclusion of abnormal scans. This suggests that our patient-based population template can serve as an alternative for identifying and quantifying perfusion abnormalities in brain SPECT.
\end{abstract}




\section{Keywords}

Brain imaging, SPECT, diagnostic imaging, cognitive impairment, traumatic brain injury, noninferiority trial, quantitative imaging biomarkers, normative database

\section{Introduction}

The application of functional neuroimaging, specifically with neuronuclear single photon emission computed tomography (SPECT), presents a number of advantages for brain diagnostics. First, brain SPECT demonstrates perfusion abnormalities (hypo or hyperperfusion) in disorders such as Alzheimer's disease (AD) [1] and traumatic brain injury (TBI) [2] that may not be evident in conventional structural imaging. Second, brain SPECT is more freely available in the office setting compared to the alternative neuronuclear modality, positron emission tomography (PET). Unlike PET, SPECT studies do not require close proximity to a cyclotron, thus improving patient access. Third, brain SPECT studies are cheaper than PET scans by at least half the cost [3]. These characteristics make SPECT an attractive modality for diagnostics of aberrant brain function.

Evaluation of any diagnostic imaging study begins with visual observations of what is different from normal. This approach proves challenging in SPECT since perfusion levels naturally vary in different regions of the brain and among different people $[4,5]$. Thus, purely visual interpretation of such scans risks negative impacts of subjectivity and interobserver variability.

The concept of quantitative analysis of brain SPECT scans has been well established for decades [6-9] but clinical grade software programs are relatively recent by comparison. Such programs are important for maximizing the utility of SPECT in clinical practice. Tools now incorporate analyses that quantify the magnitude of deviation from normal perfusion [10-13], reducing subjectivity and improving consistency and speed. The American College of Radiology (ACR) guidelines for brain SPECT reflect this shift by recommending quantitative assessment and comparison to normative datasets [5], although it is widely recognized that true values of physiologic properties like normal perfusion are impossible to measure with absolute certainty [14-16]. Normative datasets serve, at best, as surrogates for an already ambiguous truth.

SPECT normative datasets are challenging to produce [17]. Brain SPECT scans are only recommended for certain medical conditions [5] and would unnecessarily expose healthy individuals to radioactivity [18]. Other barriers include difficulty accessing a large representative sample of control subjects and the potentially prohibitive financial burden of data collection. For these reasons, companies and research groups that have invested in their own normative datasets are often reluctant to share with others who seek to quantitatively evaluate SPECT brain data. This keeps the size of commercial FDA-cleared normative datasets rather small, ranging from only 35 to 90 subjects [13,19-21], and limits competition from other groups seeking to improve SPECT quantitative tools.

We propose an alternative to traditional normative datasets by creating a "population template" that combines SPECT brain scans from a large number of clinical patients instead of a small number of healthy individuals. The outgoing impressions for these patients included TBI, toxic exposure, neuropsychiatric disorders, and others, resulting in a large heterogeneous dataset with no single defining perfusion pattern. We hypothesized that this template would prove noninferior to a smaller control dataset and therefore provide a viable substitute for use in identifying and quantifying brain perfusion abnormalities in SPECT scans.

\section{Materials and methods}

\section{Subjects}

All retrospective data analysis was done in accordance with Institutional Review Board approval (IntegReview IRB Certificate CHDB112019). In partnership with CereHealth Corporation, a total of 3, 047 of their most recent patients were assessed for inclusion in either the population template or a dataset for template validation. At the time of this study, this convenience sample was the full extent of data available for retrospective research purposes. 
All scans were acquired between the years of 2006 and 2019 from individuals across the United States and were processed according to protocols set forth by the ACR and the supervising imaging clinic, CereScan. Subjects were excluded if they had not consented their scan to be used in research $(n=424)$, if their scan data was missing $(n=188)$, if they did not complete a baseline scan $(n=26)$, if their scan was of questionable quality $(n=10)$, or if they had broken protocol $(n=4)$.

CereScan had labelled each scan with one or more outgoing diagnostic impressions derived from the reading radiologist's report. These classifications were not ground truth but permitted useful grouping of subjects. These labels were utilized to identify a dataset for template validation.

Given the existing literature on SPECT in the differential diagnosis of dementias [22-24], subjects were filtered for the following labels assigned to their scan's outgoing impressions: dementia, AD, frontotemporal dementia (FTD), cognitive impairment, and neurodegenerative processes. Subjects also had these labels if such processes could not be ruled out during the read.

The filtered dataset contained 200 scans with five duplicates. We verified each subject's inclusion by reviewing their clinical data, if available, for signs of cognitive impairment in their reported symptoms, history, and cognitive assessment scores. Due to changes in data collection over time, we found that recent patient records were consistently more complete. For this reason and resource constraints for radiologist reads, only 100 of the most recently acquired scans were chosen for inclusion in this cognitively impaired cohort (43 females and 57 males, mean age \pm SD, $63.83 \pm 11.98$ years, Table 1 ). No duplicates were included.

For the population template, the remaining set of scans were filtered to exclude subjects with gross structural abnormalities $(n=61)$, no recorded outgoing diagnostic impressions $(n=16)$, and duplicates $(n=$ 50). The final cohort of 2, 068 subjects contained 879 women and 1, 189 men with ages ranging from 4 to 83 years (mean \pm SD, $37.22 \pm 17.29$ years, Table 1 , Figure 1 ). The most common diagnostic impressions assigned to these patients were TBI $(n=1,768)$, anxiety disorder $(n=1,046)$, and $\operatorname{mood}$ disorder $(n=1,018)$ with a high incidence of comorbidity evident in the population (84\%).

Table 1. Summary characteristics about the subjects contained in MIMneuro ${ }^{\circledR} \mathrm{s}$ control dataset, the population template, and the cognitively impaired cohort. While the age range, mean, and standard deviation of MIMneuro ${ }^{\circledast \prime}$ 's controls were reported in [13], the median was not provided. The race/ethnicity of their controls were also not disclosed

\begin{tabular}{|c|c|c|c|c|}
\hline & & $\begin{array}{l}\text { MIMneuro }^{\circledR} \\
\text { controls }\end{array}$ & $\begin{array}{l}\text { Population } \\
\text { template }\end{array}$ & $\begin{array}{l}\text { Cognitively } \\
\text { impaired cohort }\end{array}$ \\
\hline \multirow[t]{3}{*}{ Age } & Mean \pm SD & $43.88 \pm 15.48$ years & $37.22 \pm 17.29$ years & $63.83 \pm 11.98$ years \\
\hline & Median & Unknown & 37 years & 65 years \\
\hline & Range & $10-89$ years & $4-83$ years & $22-87$ years \\
\hline \multirow[t]{2}{*}{ Sex } & Male & 39 & 1,189 & 57 \\
\hline & Female & 51 & 879 & 45 \\
\hline \multirow{9}{*}{$\begin{array}{l}\text { Race/ } \\
\text { Ethnicity }\end{array}$} & White & 0 & 1,493 & 78 \\
\hline & Hispanic or Latino & 0 & 125 & 1 \\
\hline & Black or African American & 0 & 57 & 3 \\
\hline & Asian & 0 & 18 & 0 \\
\hline & American Indian or Alaskan Native & 0 & 9 & 0 \\
\hline & Middle Eastern or North African & 0 & 2 & 0 \\
\hline & Native Hawaiian or other Pacific Islander & 0 & 1 & 1 \\
\hline & Two or more of the above & 0 & 50 & 1 \\
\hline & Unknown & 90 & 313 & 16 \\
\hline \multicolumn{2}{|c|}{ Total number of subjects } & 90 & 2,068 & 100 \\
\hline
\end{tabular}




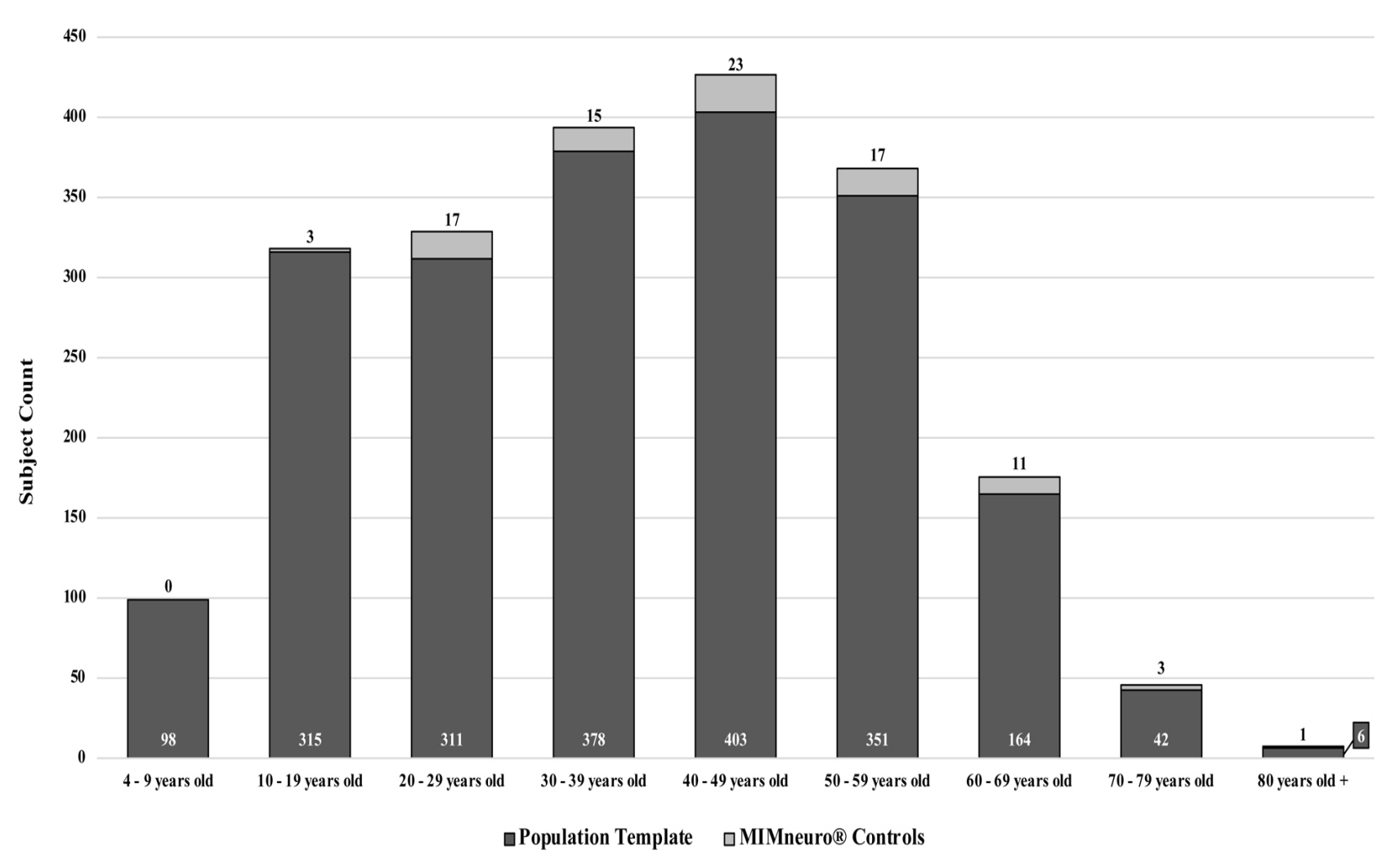

Figure 1. Age distribution of subjects in population template versus the controls contained within MIMneuro ${ }^{\circledR}$

\section{Population template generation}

All SPECT scans were performed within the guidelines set forth by the ACR [5]. Patients were instructed to abstain from certain medications for 24-48 $\mathrm{h}$ including stimulants, opiates, barbiturates, and benzodiazepines, as well as caffeine, alcohol, over-the-counter medications containing caffeine, and illicit drugs. Patients were also directed to abstain from nicotine for $10 \mathrm{~h}$ prior to the scan.

Fifteen min prior to tracer injection, each patient was made comfortable in a dimly lit and quiet room while an intravenous line was placed. The patient was asked to rest with sound dampening headphones on and their eyes closed. After $15 \mathrm{~min}$, radiopharmaceutical technetium-99m hexamethylpropyleneamine oxime (Tc99m-HMPAO) was injected into the bloodstream via the intravenous line. Uptake into the brain occurred within two min $[4,25]$. After injection, each patient rested in the same room for an additional five minutes. Approximately one hour later, the patient was placed supine on the camera bed and their head held still by a cloth strap across the forehead. Scan times varied among patients but typically lasted 20 to 30 min to collect a minimum of approximately 5 million counts.

Segami Corporation's Oasis software (Version 1.9.4.9) was used for image preprocessing. Each scan was reconstructed using filtered back projection and a Butterworth filter. Chang attenuation correction [26] was performed on all images. All raw sinograms and linograms were inspected by a nuclear medicine technologist for evidence of motion and corrected, as needed, using the built-in Cedars-Sinai MoCo application [27]. Brain volumes were then manually masked with ellipses by a nuclear medicine technologist to exclude non-neural uptake.

After masking, each reconstructed volume was sent through CereMetrix ${ }^{\circledR}$ software (Version 1.0) for registration. This software first thresholded the volume using Otsu's method [28]. The original and thresholded versions were then mapped to the Montreal Neurological Institute (MNI) standard single subject brain template [29] using an affine transformation with 12 degrees of freedom. The optimal transformation for each volume was determined using the advanced mattes mutual information metric (MI) and adaptive 
stochastic gradient decent. The transformation with the higher MI was then applied to the original data and image intensities were normalized to the average intensity value of the whole brain [30].

After processing, all scans were exported and a script averaged the intensity values of each voxel across all scans to compute the population template. That is, every voxel in the template represented an average of 2, 068 normalized intensity values at the same location in each scan (Figure 2).

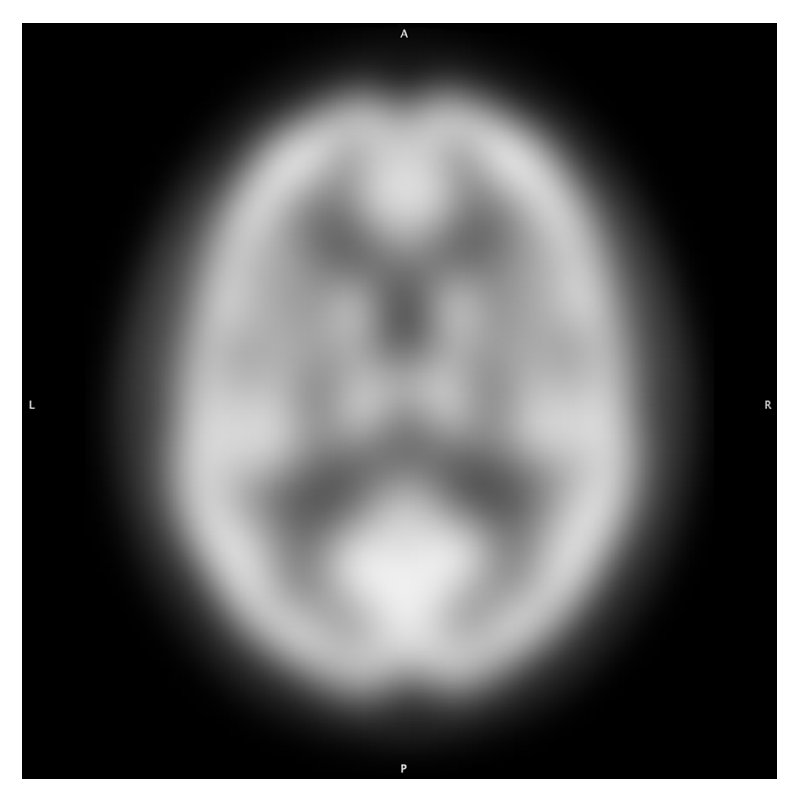

Figure 2. Axial cross section of the population template generated from 2, 068 clinical scans acquired at standard SPECT resolution and processed as described for display in the MNI single subject space

\section{Template comparison to normative database}

Ideally, the population template would be evaluated against true values or a reference standard, such as a phantom or digital reference object, to assess the technical performance associated with its use in quantifying brain perfusion abnormalities [31]. To our knowledge, no publicly available physical or digital SPECT reference standard has been widely accepted to model normal cerebral perfusion [15]. Therefore, validation of the population template is restricted to comparisons against softwares containing normative databases.

For this study, the population template was imported into quantitative SPECT brain analysis software, MIMneuro $^{\circledR}$ (Version 6.7.12), and mathematically compared against its normative dataset through their region and cluster analysis tools. This is one of the largest control datasets in commercial software, containing Tc99m-HMPAO SPECT brain scans from 90 healthy volunteers ( 51 females and 39 males, mean age $=43.88 \pm 15.48$ years, Table 1, Figure 1) $[13,19]$. A custom MIMneuro ${ }^{\circledR}$ workflow was built to import the template, normalize voxel intensities to the mean value for the whole brain, register the template to its proprietary brain atlas, and identify regions of interest (ROI) at varying levels of anatomical detail. MIMneuro $^{\circledR}$ 's registration tool, BrainAlign ${ }^{\mathrm{TM}}$, has been previously described [32]. The workflow then compared each voxel of the template to the same voxel in the controls. A z-score was calculated at every voxel to quantify its difference from the controls and generate a z-score map. Voxel z-scores were then aggregated and averaged according to their anatomical region assignment and 95\% confidence intervals (CIs) were calculated and corrected for multiple comparisons using the conservative Bonferroni method [33, 34]. The region results were assessed for noninferiority using MIMneuro ${ }^{\circledR \prime}$ 's default threshold for normality, 1.65 z-score, as the noninferiority margin [13]. CIs within this margin would indicate that the population template was noninferior to MIMneuro ${ }^{\circledR \prime}$ ' normative dataset [31].

MIMneuro ${ }^{\circledR}$ 's default voxel analysis was then used to identify clusters of abnormal voxels that may span region borders. The following parameters dictated how the software identified clusters: $P$-value, minimum volume, and minimum z-score. The $P$-value determined the statistical significance level of the cluster, or the likelihood that a cluster was truly abnormal rather than occurring by chance [13]. The $P$-value parameter was 
set to one to identify all clusters, regardless of statistical significance. The minimum volume parameter, which defined the minimum size of the cluster in milliliters, was set to zero to return clusters of any size. Finally, only voxels that met the minimum z-score requirement were eligible for clustering. We set this parameter to $1.65 \mathrm{z}$-score to correspond to MIMneuro ${ }^{\circledR}$ 's default threshold for normality and our noninferiority margin.

\section{ROI analysis against template and normative database}

The template and controls are both intended to serve as comparison datasets for quantifying deviations from typical perfusion in individual scans. However, each software deploys proprietary processing prior to this calculation that could impact the final output of perfusion measurements even if the population template was found to be sufficiently close to MIMneuro ${ }^{\circledR}$ 's normative. Therefore, assessing agreement between the z-score data generated from the template and controls within their respective softwares would further support whether measurements made from the template are noninferior to those made from the normative dataset.

The population template was integrated into a research-only instance of the CereMetrix ${ }^{\circledR}$ image processing pipeline that performed registration and normalization of image voxel values (previously described in "Population template generation" section) and calculated a z-score at every voxel to quantify its difference from the population template. This pipeline also designated an anatomical label for each voxel corresponding to a region contained within the automatic anatomical labeling atlas (AAL) [35].

The cohort of 100 cognitively impaired subjects was used to compare z-score results generated by CereMetrix $^{\circledR}$ using the population template to those by MIMneuro $^{\circledR}$ using its controls. Their scans were acquired, reconstructed, and motion corrected in Oasis software and then separately processed in both CereMetrix $^{\circledR}$ and MIMneuro ${ }^{\circledR}$ for spatial registration, intensity normalization, and z-score calculation, as previously described. All average region z-scores were exported for analysis in Python $^{\text {TM }}$ or Microsoft ${ }^{\circledR}$ Excel. $^{-}$

Since the regions identified by each software were not identical due to registration and atlas differences, smaller regions were grouped into left and right internal or lobar (frontal, temporal, parietal) structures (Table 2). MIMneuro ${ }^{\circledR}$ automatically addresses this issue by offering the lobar structures as individual regions, but it does not do so for internal structures. Instead, a script was written to average the average z-scores for these regions from both MIMneuro ${ }^{\circledR}$ and CereMetrix ${ }^{\circledR}$ data. However, to generate data for CereMetrix ${ }^{\circledR}$ lobar structures consistently with MIMneuro ${ }^{\circledR}$, the script only performed one average across the voxels of the smaller structures. This resulted in eight measurements per patient for a final total of 800 measurements from each software.

Agreement metrics often assume that the data are normally distributed. All data and their differences between softwares were graphed on probability plots to confirm this prior to the calculation of the following scaled and unscaled agreement indices: concordance and intraclass correlation coefficients [CCC, ICC $(3,1)]$ [36, 37], mean squared deviations (MSD) [31], total deviation indices (TDI) [38], and Bland-Altman plots with limits of agreement (LOA) [39, 40]. 95\% CIs were calculated for each metric [34, 36], corrected for multiple comparisons using the Bonferroni method [33], and, where appropriate, compared against the noninferiority margin.

\section{Visual reads after template comparison}

Despite the objectivity and standardization that region-based quantitative metrics bring to brain SPECT reads, the spatial distribution of perfusion abnormalities is limited when voxel z-scores are averaged across ROIs. It is the spatial distribution of voxel z-scores that is most utilized by physicians performing individual brain SPECT reads, not ROI-averaged metrics. Agreement between softwares, present or not, bears no merit on the clinical utility of the population template if established voxel-wise perfusion patterns do not appear. Accurate reads are dependent on them.

To assess this, a single reader (CR) with extensive experience in reading Tc99m-HMPAO SPECT brain scans was trained on the CereMetrix ${ }^{\circledR}$ platform and given access the clinical and imaging data for each deidentified subject in the cognitively impaired cohort. This reader categorized his observed impressions of perfusion 
patterns as normal, AD, FTD, Lewy body dementia, TBI, vascular dementia, mixed dementia, indeterminate, or other psychiatric pattern. These categorizations were then compared with the original impressions generated by six readers using other SPECT analysis platforms. Concordance was evaluated in Microsoft ${ }^{\circledR}$ Excel using Krippendorff's alpha statistic for multiple raters with 95\% CIs [41, 42] and interpreted like other kappa statistics: 0.0-0.2 as slight agreement, 0.21-0.4 as fair agreement, 0.41-0.6 as moderate agreement, 0.61-0.8 as substantial agreement, and over 0.8 as nearly perfect agreement [43]. Substantial to near perfect agreement between readers' impressions, despite the use of different softwares and comparison datasets, would support the clinical utility of the population template for individual patient analysis.

Table 2. Summary of regions that were aggregated to create four metrics for each patient of the cognitively impaired cohort

\begin{tabular}{|c|c|c|}
\hline & MIMneuro $^{\circledR}$ & CereMetrix $^{\circledR}$ \\
\hline \multirow[t]{15}{*}{ Frontal lobe } & Frontal lobe & Anterior orbital gyrus \\
\hline & & Gyrus rectus \\
\hline & & Inferior frontal gyrus, pars opercularis \\
\hline & & Inferior frontal gyrus, pars orbitalis \\
\hline & & Inferior frontal gyrus, pars triangularis \\
\hline & & Lateral orbital gyrus \\
\hline & & Medial orbital gyrus \\
\hline & & Medial orbitofrontal cortex \\
\hline & & Middle frontal gyrus \\
\hline & & Olfactory cortex \\
\hline & & Posterior orbital gyrus \\
\hline & & Precentral gyrus \\
\hline & & Superior frontal gyrus \\
\hline & & Superior frontal gyrus, medial part \\
\hline & & Supplementary motor area \\
\hline \multirow[t]{6}{*}{ Parietal lobe } & Parietal lobe & Angular gyrus \\
\hline & & Inferior parietal lobule \\
\hline & & Postcentral gyrus \\
\hline & & Precuneus \\
\hline & & Superior parietal lobule \\
\hline & & Supramarginal gyrus \\
\hline \multirow[t]{7}{*}{ Temporal lobe } & Temporal lobe & Fusiform gyrus \\
\hline & & Heschl's gyrus \\
\hline & & Inferior temporal gyrus \\
\hline & & Middle temporal gyrus \\
\hline & & Middle temporal pole \\
\hline & & Superior temporal gyrus \\
\hline & & Superior temporal pole \\
\hline \multirow[t]{10}{*}{ Internal structures } & Amygdala & Amygdala \\
\hline & Caudate & Anterior cingulate gyrus \\
\hline & Cingulate gyrus & Caudate \\
\hline & Globus pallidus & Globus pallidus \\
\hline & Hippocampus & Hippocampus \\
\hline & Parahippocampal gyrus & Middle cingulate gyrus \\
\hline & Putamen & Parahippocampal gyrus \\
\hline & Thalamus & Posterior cingulate gyrus \\
\hline & & Putamen \\
\hline & & Thalamus \\
\hline
\end{tabular}




\section{Results}

Template comparison to normative database

The average z-score deviations from the controls for each region of MIMneuro ${ }^{\circledR}$ 's atlas are given in Table 3 while their summary statistics and histogram of their distribution are in Figure 3. No regions of the population template fell outside $\pm 1 \mathrm{z}$-score, $84 \%$ were within \pm 0.5 z-score, and no CIs exceeded the noninferiority margin of $\pm 1.65 \mathrm{z}$-score.

Voxel analysis produced nine clusters that met the criteria described previously (Table 4). The largest cluster $(11.53 \mathrm{~mL})$ had an average z-score of 1.9 and spanned areas of the left lateral temporal and occipital lobes (Figure 4). This cluster and all others had low statistical significance $(P \geq 0.93)$.

Table 3. Mean z-scores and 95\% Cls quantifying the population template's deviation from MIMneuro ${ }^{\circledR}$ 's controls for every region in MIMneuro ${ }^{\circledR}$ 's proprietary atlas

\begin{tabular}{|c|c|c|c|c|c|c|c|}
\hline Region & $\begin{array}{l}\text { Mean } \\
{[95 \% \mathrm{CI}]}\end{array}$ & Region & $\begin{array}{l}\text { Mean } \\
{[95 \% \mathrm{Cl}]}\end{array}$ & Region & $\begin{array}{l}\text { Mean } \\
{[95 \% \mathrm{Cl}]}\end{array}$ & Region & $\begin{array}{l}\text { Mean } \\
{[95 \% \mathrm{Cl}]}\end{array}$ \\
\hline Amygdala (L) & $\begin{array}{l}-0.28 \\
{[-0.34,-0.22]}\end{array}$ & $\begin{array}{l}\text { Inf. frontal } \\
\text { gyrus (L) }\end{array}$ & $\begin{array}{l}-0.43 \\
{[-0.45,-0.41]}\end{array}$ & $\begin{array}{l}\text { Middle orbital } \\
\text { gyrus (L) }\end{array}$ & $\begin{array}{l}-0.09 \\
{[-0.11,-0.07]}\end{array}$ & $\begin{array}{l}\text { Retrosplenial } \\
\text { area }(\mathrm{L})\end{array}$ & $\begin{array}{l}0.09 \\
{[0.02,0.16]}\end{array}$ \\
\hline Amygdala (R) & $\begin{array}{l}-0.84 \\
{[-0.93,-0.75]}\end{array}$ & $\begin{array}{l}\text { Inf. frontal } \\
\text { gyrus (R) }\end{array}$ & $\begin{array}{l}-0.45 \\
{[-0.47,-0.43]}\end{array}$ & $\begin{array}{l}\text { Middle orbital } \\
\text { gyrus }(R)\end{array}$ & $\begin{array}{l}-0.31 \\
{[-0.37,-0.25]}\end{array}$ & $\begin{array}{l}\text { Retrosplenial } \\
\text { area }(R)\end{array}$ & $\begin{array}{l}-0.06 \\
{[-0.10,-0.02]}\end{array}$ \\
\hline $\begin{array}{l}\text { Angular gyrus } \\
\text { (L) }\end{array}$ & $\begin{array}{l}0.33 \\
{[0.31,0.35]}\end{array}$ & $\begin{array}{l}\text { Inf. frontal } \\
\text { gyrus, pars } \\
\text { opercularis (L) }\end{array}$ & $\begin{array}{l}-0.57 \\
{[-0.61,-0.52]}\end{array}$ & $\begin{array}{l}\text { Middle } \\
\text { temporal gyrus } \\
\text { (L) }\end{array}$ & $\begin{array}{l}0.49 \\
{[0.46,0.52]}\end{array}$ & $\begin{array}{l}\text { Rolandic } \\
\text { operculum (L) }\end{array}$ & $\begin{array}{l}-0.37 \\
{[-0.40,-0.34]}\end{array}$ \\
\hline $\begin{array}{l}\text { Angular gyrus } \\
(\mathrm{R})\end{array}$ & $\begin{array}{l}0.13 \\
{[0.11,0.15]}\end{array}$ & $\begin{array}{l}\text { Inf. frontal } \\
\text { gyrus, pars } \\
\text { opercularis (R) }\end{array}$ & $\begin{array}{l}-0.59 \\
{[-0.62,-0.56]}\end{array}$ & $\begin{array}{l}\text { Middle } \\
\text { temporal gyrus } \\
\text { (R) }\end{array}$ & $\begin{array}{l}0.46 \\
{[0.43,0.49]}\end{array}$ & $\operatorname{lic}$ lum (R) & $\begin{array}{l}-0.55 \\
{[-0.59,-0.51]}\end{array}$ \\
\hline $\begin{array}{l}\text { Anterior } \\
\text { cingulate gyrus } \\
\text { (L) }\end{array}$ & $\begin{array}{l}0.15 \\
{[0.13,0.17]}\end{array}$ & $\begin{array}{l}\text { Inf. frontal } \\
\text { gyrus, pars } \\
\text { orbitalis (L) }\end{array}$ & $\begin{array}{l}-0.37 \\
{[-0.42,-0.32]}\end{array}$ & $\begin{array}{l}\text { Nucleus } \\
\text { accumbens (L) }\end{array}$ & $\begin{array}{l}-0.04 \\
{[-0.07,-0.01]}\end{array}$ & losal & $\begin{array}{l}-0.12 \\
{[-0.20,-0.04]}\end{array}$ \\
\hline $\begin{array}{l}\text { Anterior } \\
\text { cingulate gyrus } \\
\text { (R) }\end{array}$ & $\begin{array}{l}0.22 \\
{[0.19,0.25]}\end{array}$ & $\begin{array}{l}\text { Inf. frontal } \\
\text { gyrus, pars } \\
\text { orbitalis (R) }\end{array}$ & $\begin{array}{l}-0.25 \\
{[-0.31,-0.19]}\end{array}$ & $\begin{array}{l}\text { Nucleus } \\
\text { accumbens (R) }\end{array}$ & $\begin{array}{l}-0.33 \\
{[-0.43,-0.23]}\end{array}$ & $\begin{array}{l}\text { llosal } \\
\text { २) }\end{array}$ & $\begin{array}{l}-0.13 \\
{[-0.21,-0.05]}\end{array}$ \\
\hline $\begin{array}{l}\text { Anterior orbital } \\
\text { gyrus (L) }\end{array}$ & $\begin{array}{l}-0.03 \\
{[-0.05,-0.01]}\end{array}$ & $\begin{array}{l}\text { Inf. frontal } \\
\text { gyrus, pars } \\
\text { triangularis (L) }\end{array}$ & $\begin{array}{l}-0.32 \\
{[-0.35,-0.29]}\end{array}$ & $\begin{array}{l}\text { Occipital lobe } \\
\text { (L) }\end{array}$ & $\begin{array}{l}0.56 \\
{[0.54,0.58]}\end{array}$ & $\begin{array}{l}\text { rebellar } \\
\text { le }(L)\end{array}$ & $\begin{array}{l}0.18 \\
{[0.09,0.27]}\end{array}$ \\
\hline $\begin{array}{l}\text { Anterior orbital } \\
\text { gyrus }(R)\end{array}$ & $\begin{array}{l}-0.12 \\
{[-0.20,-0.04]}\end{array}$ & $\begin{array}{l}\text { Inf. frontal } \\
\text { gyrus, pars } \\
\text { triangularis (R) }\end{array}$ & $\begin{array}{l}-0.41 \\
{[-0.45,-0.37]}\end{array}$ & $\begin{array}{l}\text { Occipital lobe } \\
\text { (R) }\end{array}$ & $\begin{array}{l}0.32 \\
{[0.31,0.33]}\end{array}$ & $\begin{array}{l}\text { cerebellar } \\
\text { cle }(R)\end{array}$ & $\begin{array}{l}0.22 \\
{[0.15,0.29]}\end{array}$ \\
\hline Basis & $\begin{array}{l}0.06 \\
{[0.05,0.07]}\end{array}$ & $\begin{array}{l}\text { Inf. medial } \\
\text { frontal gyrus } \\
\text { (L) }\end{array}$ & $\begin{array}{l}-0.51 \\
{[-0.55,-0.47]}\end{array}$ & $\begin{array}{l}\text { Olfactory } \\
\text { cortex (L) }\end{array}$ & $\begin{array}{l}-0.04 \\
{[-0.06,-0.02]}\end{array}$ & $\begin{array}{l}\text { Sup. frontal } \\
\text { gyrus }(L)\end{array}$ & $\begin{array}{l}0.26 \\
{[0.24,0.28]}\end{array}$ \\
\hline Brair & $\begin{array}{l}0.07 \\
{[0.06,0.08]}\end{array}$ & $\begin{array}{l}\text { Inf. medial } \\
\text { frontal gyrus } \\
(\mathrm{R})\end{array}$ & $\begin{array}{l}-0.86 \\
{[-0.91,-0.81]}\end{array}$ & $\begin{array}{l}\text { Olfactory } \\
\text { cortex (R) }\end{array}$ & $\begin{array}{l}-0.42 \\
{[-0.57,-0.27]}\end{array}$ & $\begin{array}{l}\text { Sup. frontal } \\
\text { gyrus (R) }\end{array}$ & $\begin{array}{l}0.26 \\
{[0.24,0.28]}\end{array}$ \\
\hline Caudate (L) & $\begin{array}{l}-0.10 \\
{[-0.13,-0.07]}\end{array}$ & $\begin{array}{l}\text { Inf. occipital } \\
\text { gyrus (L) }\end{array}$ & $\begin{array}{l}0.48 \\
{[0.43,0.53]}\end{array}$ & $\begin{array}{l}\text { Orbitofrontal } \\
\text { region (L) }\end{array}$ & $\begin{array}{l}-0.18 \\
{[-0.29,-0.16]}\end{array}$ & $\begin{array}{l}\text { Sup. medial } \\
\text { frontal gyrus } \\
\text { (L) }\end{array}$ & $\begin{array}{l}-0.11 \\
{[-0.13,-0.09]}\end{array}$ \\
\hline Cauc & $\begin{array}{l}-0.15 \\
{[-0.20,-0.10]}\end{array}$ & $\begin{array}{l}\text { Inf. occipital } \\
\text { gyrus (R) }\end{array}$ & $\begin{array}{l}0.28 \\
{[0.24,0.32]}\end{array}$ & $\begin{array}{l}\text { Orbitofrontal } \\
\text { region }(R)\end{array}$ & $\begin{array}{l}-0.41 \\
{[-0.45,-0.37]}\end{array}$ & $\begin{array}{l}\text { Sup. medial } \\
\text { frontal gyrus } \\
\text { (R) }\end{array}$ & $\begin{array}{l}-0.13 \\
{[-0.15,-0.11]}\end{array}$ \\
\hline $\begin{array}{l}\text { Cerebellar } \\
\text { hemisphere }(\mathrm{L})\end{array}$ & $\begin{array}{l}0.59 \\
{[0.57,0.61]}\end{array}$ & $\begin{array}{l}\text { Inf. temporal } \\
\text { gyrus (L) }\end{array}$ & $\begin{array}{l}0.63 \\
{[0.59,0.67]}\end{array}$ & $\begin{array}{l}\text { Paracentral } \\
\text { lobule (L) }\end{array}$ & $\begin{array}{l}-0.17 \\
{[-0.19,-0.15]}\end{array}$ & $\begin{array}{l}\text { Sup. occipital } \\
\text { gyrus (L) }\end{array}$ & $\begin{array}{l}0.53 \\
{[0.50,0.56]}\end{array}$ \\
\hline $\begin{array}{l}\text { Cerebellar } \\
\text { hemisphere (R) }\end{array}$ & $\begin{array}{l}0.29 \\
{[0.28,0.30]}\end{array}$ & $\begin{array}{l}\text { Inf. temporal } \\
\text { gyrus }(R)\end{array}$ & $\begin{array}{l}0.11 \\
{[0.10,0.12]}\end{array}$ & $\begin{array}{l}\text { Paracentral } \\
\text { lobule }(R)\end{array}$ & $\begin{array}{l}-0.12 \\
{[-0.14,-0.10]}\end{array}$ & $\begin{array}{l}\text { Sup. occipital } \\
\text { gyrus }(R)\end{array}$ & $\begin{array}{l}0.51 \\
{[0.48,0.54]}\end{array}$ \\
\hline $\begin{array}{l}\text { Cerebellar } \\
\text { vermis }\end{array}$ & $\begin{array}{l}0.51 \\
{[0.48,0.54]}\end{array}$ & Insula (L) & $\begin{array}{l}-0.35 \\
{[-0.38,-0.32]}\end{array}$ & $\begin{array}{l}\text { Parahippo- } \\
\text { campal gyrus } \\
\text { (L) }\end{array}$ & $\begin{array}{l}0.20 \\
{[0.16,0.24]}\end{array}$ & $\begin{array}{l}\text { Sup. parietal } \\
\text { lobule (L) }\end{array}$ & $\begin{array}{l}0.24 \\
{[0.22,0.26]}\end{array}$ \\
\hline
\end{tabular}


Table 3. Mean z-scores and $95 \%$ Cls quantifying the population template's deviation from MIMneuro ${ }^{\circledR}$ 's controls for every region in MIMneuro ${ }^{\circledast \prime}$ 's proprietary atlas (continued)

\begin{tabular}{|c|c|c|c|c|c|c|c|}
\hline Region & $\begin{array}{l}\text { Mean } \\
{[95 \% \mathrm{Cl}]}\end{array}$ & Region & $\begin{array}{l}\text { Mean } \\
{[95 \% \mathrm{Cl}]}\end{array}$ & Region & $\begin{array}{l}\text { Mean } \\
{[95 \% \mathrm{Cl}]}\end{array}$ & Region & $\begin{array}{l}\text { Mean } \\
{[95 \% \mathrm{Cl}]}\end{array}$ \\
\hline Cerebellum & $\begin{array}{l}0.45 \\
{[0.44,0.46]}\end{array}$ & Incol & $\begin{array}{l}-0.83 \\
{[-0.87,-0.79]}\end{array}$ & $\begin{array}{l}\text { Parahippo- } \\
\text { campal gyrus } \\
(\mathrm{R})\end{array}$ & $\begin{array}{l}-0.20 \\
{[-0.23,-0.17]}\end{array}$ & $\begin{array}{l}\text { Sup. parietal } \\
\text { lobule }(R)\end{array}$ & $\begin{array}{l}0.12 \\
{[0.10,0.14]}\end{array}$ \\
\hline $\begin{array}{l}\text { Cingulate gyrus } \\
\text { (L) }\end{array}$ & $\begin{array}{l}0.09 \\
{[0.07,0.11]}\end{array}$ & $\begin{array}{l}\text { Lateral orbital } \\
\text { gyrus (L) }\end{array}$ & $\begin{array}{l}-0.12 \\
{[-0.15,-0.09]}\end{array}$ & $\begin{array}{l}\text { Parietal lobe } \\
\text { (L) }\end{array}$ & $\begin{array}{l}0.15 \\
{[0.14,0.16]}\end{array}$ & $\begin{array}{l}\text { Sup. temporal } \\
\text { gyrus (L) }\end{array}$ & $\begin{array}{l}0.34 \\
{[0.30,0.38]}\end{array}$ \\
\hline $\begin{array}{l}\text { Cingulate gyrus } \\
(R)\end{array}$ & $\begin{array}{l}0.08 \\
{[0.06,0.10]}\end{array}$ & $\begin{array}{l}\text { Lateral orbital } \\
\text { gyrus }(R)\end{array}$ & $\begin{array}{l}-0.35 \\
{[-0.42,-0.28]}\end{array}$ & $\begin{array}{l}\text { Parietal lobe } \\
(R)\end{array}$ & $\begin{array}{l}-0.03 \\
{[-0.04,-0.02]}\end{array}$ & $\begin{array}{l}\text { Sup. temporal } \\
\text { gyrus }(R)\end{array}$ & $\begin{array}{l}0.13 \\
{[0.09,0.17]}\end{array}$ \\
\hline Cuneus (L) & $\begin{array}{l}0.23 \\
{[0.20,0.26]}\end{array}$ & $\begin{array}{l}\text { Lateral } \\
\text { temporal lobe } \\
\text { (L) }\end{array}$ & $\begin{array}{l}0.44 \\
{[0.42,0.46]}\end{array}$ & Pons & $\begin{array}{l}0.08 \\
{[0.07,0.09]}\end{array}$ & $\begin{array}{l}\text { Supplementary } \\
\text { motor area (L) }\end{array}$ & $\begin{array}{l}-0.03 \\
{[-0.05,-0.01]}\end{array}$ \\
\hline Cuneus (R) & $\begin{array}{l}0.2 \\
{[0.17,0.23]}\end{array}$ & $\begin{array}{l}\text { Lateral } \\
\text { temporal lobe } \\
(\mathrm{R})\end{array}$ & $\begin{array}{l}0.25 \\
{[0.23,0.27]}\end{array}$ & ie & $\begin{array}{l}0.14 \\
{[0.12,0.16]}\end{array}$ & $\begin{array}{l}\text { tary } \\
\text { (R) }\end{array}$ & $\begin{array}{l}-0.01 \\
{[-0.02,0.00]}\end{array}$ \\
\hline Frontal lobe (L) & $\begin{array}{l}-0.02 \\
{[-0.03,-0.01]}\end{array}$ & $\begin{array}{l}\text { Lingual gyrus } \\
\text { (L) }\end{array}$ & $\begin{array}{l}0.65 \\
{[0.61,0.69]}\end{array}$ & tral & $\begin{array}{l}0.18 \\
{[0.16,0.20]}\end{array}$ & arginal & $\begin{array}{l}0.31 \\
{[0.29,0.33]}\end{array}$ \\
\hline Fron & $\begin{array}{l}-0.08 \\
{[-0.09,-0.07]}\end{array}$ & $\begin{array}{l}\text { Lingual gyrus } \\
(\mathrm{R})\end{array}$ & $\begin{array}{l}0.13 \\
{[0.11,0.15]}\end{array}$ & $\begin{array}{l}\text { Postcentral } \\
\text { gyrus (R) }\end{array}$ & $\begin{array}{l}-0.03 \\
{[-0.05,-0.01]}\end{array}$ & $\begin{array}{l}\text { larginal } \\
\text { २) }\end{array}$ & $\begin{array}{l}-0.05 \\
{[-0.08,-0.02]}\end{array}$ \\
\hline $\begin{array}{l}\text { Fusiform gyrus } \\
\text { (L) }\end{array}$ & $\begin{array}{l}0.76 \\
{[0.73,0.79]}\end{array}$ & $\begin{array}{l}\text { Medial orbital } \\
\text { gyrus (L) }\end{array}$ & $\begin{array}{l}-0.29 \\
{[-0.33,-0.25]}\end{array}$ & $\begin{array}{l}\text { Posterior } \\
\text { cingulate gyrus } \\
\text { (L) }\end{array}$ & $\begin{array}{l}-0.01 \\
{[-0.03,0.01]}\end{array}$ & $\begin{array}{l}\text { Temporal lobe } \\
\text { (L) }\end{array}$ & $\begin{array}{l}0.39 \\
{[0.37,0.41]}\end{array}$ \\
\hline $\begin{array}{l}\text { Fusiform gyrus } \\
\text { (R) }\end{array}$ & $\begin{array}{l}0.08 \\
{[0.07,0.09]}\end{array}$ & rbital & $\begin{array}{l}-0.54 \\
{[-0.61,-0.47]}\end{array}$ & $\begin{array}{l}\text { Posterior } \\
\text { cingulate gyrus } \\
\text { (R) }\end{array}$ & $\begin{array}{l}0.00 \\
{[-0.03,0.03]}\end{array}$ & $\begin{array}{l}\text { Temporal lobe } \\
(\mathrm{R})\end{array}$ & $\begin{array}{l}0.10 \\
{[0.09,0.11]}\end{array}$ \\
\hline $\begin{array}{l}\text { Globus pallidus } \\
\text { (L) }\end{array}$ & $\begin{array}{l}0.04 \\
{[-0.01,0.09]}\end{array}$ & $\begin{array}{l}\text { Medial } \\
\text { temporal lobe } \\
\text { (L) }\end{array}$ & $\begin{array}{l}0.17 \\
{[0.14,0.20]}\end{array}$ & $\begin{array}{l}\text { Posterior } \\
\text { orbital gyrus (L) }\end{array}$ & $\begin{array}{l}-0.17 \\
{[-0.20,-0.14]}\end{array}$ & $\begin{array}{l}\text { Temporal } \\
\text { operculum (L) }\end{array}$ & $\begin{array}{l}-0.57 \\
{[-0.62,-0.52]}\end{array}$ \\
\hline $\begin{array}{l}\text { Globus pallidus } \\
\text { (R) }\end{array}$ & $\begin{array}{l}-0.3 \\
{[-0.35,-0.25]}\end{array}$ & $\begin{array}{l}\text { Medial } \\
\text { temporal lobe } \\
(\mathrm{R})\end{array}$ & $\begin{array}{l}-0.28 \\
{[-0.31,-0.25]}\end{array}$ & $\begin{array}{l}\text { Posterior } \\
\text { orbital gyrus } \\
(\mathrm{R})\end{array}$ & $\begin{array}{l}-0.59 \\
{[-0.68,-0.50]}\end{array}$ & $\begin{array}{l}\text { Temporal } \\
\text { operculum (R) }\end{array}$ & $\begin{array}{l}-0.57 \\
{[-0.63,-0.51]}\end{array}$ \\
\hline $\begin{array}{l}\text { Gyrus rectus } \\
\text { (L) }\end{array}$ & $\begin{array}{l}-0.40 \\
{[-0.49,-0.31]}\end{array}$ & Medulla & $\begin{array}{l}0.11 \\
{[0.08,0.14]}\end{array}$ & $\begin{array}{l}\text { Precentral } \\
\text { gyrus (L) }\end{array}$ & $\begin{array}{l}0.23 \\
{[0.20,0.26]}\end{array}$ & $\begin{array}{l}\text { Temporal pole } \\
\text { (L) }\end{array}$ & $\begin{array}{l}-0.27 \\
{[-0.31,-0.23]}\end{array}$ \\
\hline $\begin{array}{l}\text { Gyrus rectus } \\
\text { (R) }\end{array}$ & $\begin{array}{l}-0.46 \\
{[-0.56,-0.36]}\end{array}$ & Midb & $\begin{array}{l}0.04 \\
{[0.02,0.06]}\end{array}$ & $\begin{array}{l}\text { Precentral } \\
\text { gyrus (R) }\end{array}$ & $\begin{array}{l}0.07 \\
{[0.04,0.10]}\end{array}$ & $\begin{array}{l}\text { Temporal pole } \\
(\mathrm{R})\end{array}$ & $\begin{array}{l}-0.40 \\
{[-0.44,-0.36]}\end{array}$ \\
\hline $\begin{array}{l}\text { Heschl gyrus } \\
\text { (L) }\end{array}$ & $\begin{array}{l}0.03 \\
{[-0.02,0.08]}\end{array}$ & $\begin{array}{l}\text { Middle } \\
\text { cerebellar } \\
\text { peduncle (L) }\end{array}$ & $\begin{array}{l}0.01 \\
{[0.00,0.02]}\end{array}$ & (L) & $\begin{array}{l}-0.21 \\
{[-0.23,-0.19]}\end{array}$ & (L) & $\begin{array}{l}0.70 \\
{[0.66,0.74]}\end{array}$ \\
\hline $\begin{array}{l}\text { Heschl gyrus } \\
(\mathrm{R})\end{array}$ & $\begin{array}{l}-0.32 \\
{[-0.39,-0.25]}\end{array}$ & $\begin{array}{l}\text { Middle } \\
\text { cerebellar } \\
\text { peduncle }(\mathrm{R})\end{array}$ & $\begin{array}{l}0.01 \\
{[0.00,0.02]}\end{array}$ & Precuneus $(R)$ & $\begin{array}{l}-0.23 \\
{[-0.25,-0.21]}\end{array}$ & (R) & $\begin{array}{l}0.47 \\
{[0.43,0.51]}\end{array}$ \\
\hline $\begin{array}{l}\text { Hippocampus } \\
\text { (L) }\end{array}$ & $\begin{array}{l}0.11 \\
{[0.06,0.16]}\end{array}$ & $\begin{array}{l}\text { Middle frontal } \\
\text { gyrus (L) }\end{array}$ & $\begin{array}{l}0.21 \\
{[0.19,0.23]}\end{array}$ & $\begin{array}{l}\text { Primary visual } \\
\text { cortex }(\mathrm{L})\end{array}$ & $\begin{array}{l}0.42 \\
{[0.39,0.45]}\end{array}$ & Whole brain & $\begin{array}{l}0.10 \\
{[0.10,0.10]}\end{array}$ \\
\hline $\begin{array}{l}\text { Hippocampus } \\
\text { (R) }\end{array}$ & $\begin{array}{l}-0.45 \\
{[-0.50,-0.40]}\end{array}$ & $\begin{array}{l}\text { Middle frontal } \\
\text { gyrus (R) }\end{array}$ & $\begin{array}{l}0.41 \\
{[0.39,0.43]}\end{array}$ & $\begin{array}{l}\text { Primary visual } \\
\text { cortex }(R)\end{array}$ & $\begin{array}{l}0.35 \\
{[0.32,0.38]}\end{array}$ & & \\
\hline $\begin{array}{l}\text { Inf. cerebellar } \\
\text { peduncle (L) }\end{array}$ & $\begin{array}{l}0.11 \\
{[-0.10,0.32]}\end{array}$ & $\begin{array}{l}\text { Middle occipital } \\
\text { gyrus (L) }\end{array}$ & $\begin{array}{l}0.72 \\
{[0.68,0.76]}\end{array}$ & Putamen (L) & $\begin{array}{l}0.07 \\
{[0.05,0.09]}\end{array}$ & & \\
\hline $\begin{array}{l}\text { Inf. cerebellar } \\
\text { peduncle }(\mathrm{R})\end{array}$ & $\begin{array}{l}0.06 \\
{[-0.10,0.22]}\end{array}$ & $\begin{array}{l}\text { Middle occipital } \\
\text { gyrus }(R)\end{array}$ & $\begin{array}{l}0.37 \\
{[0.35,0.39]}\end{array}$ & Putamen (R) & $\begin{array}{l}-0.47 \\
{[-0.51,-0.43]}\end{array}$ & & \\
\hline
\end{tabular}




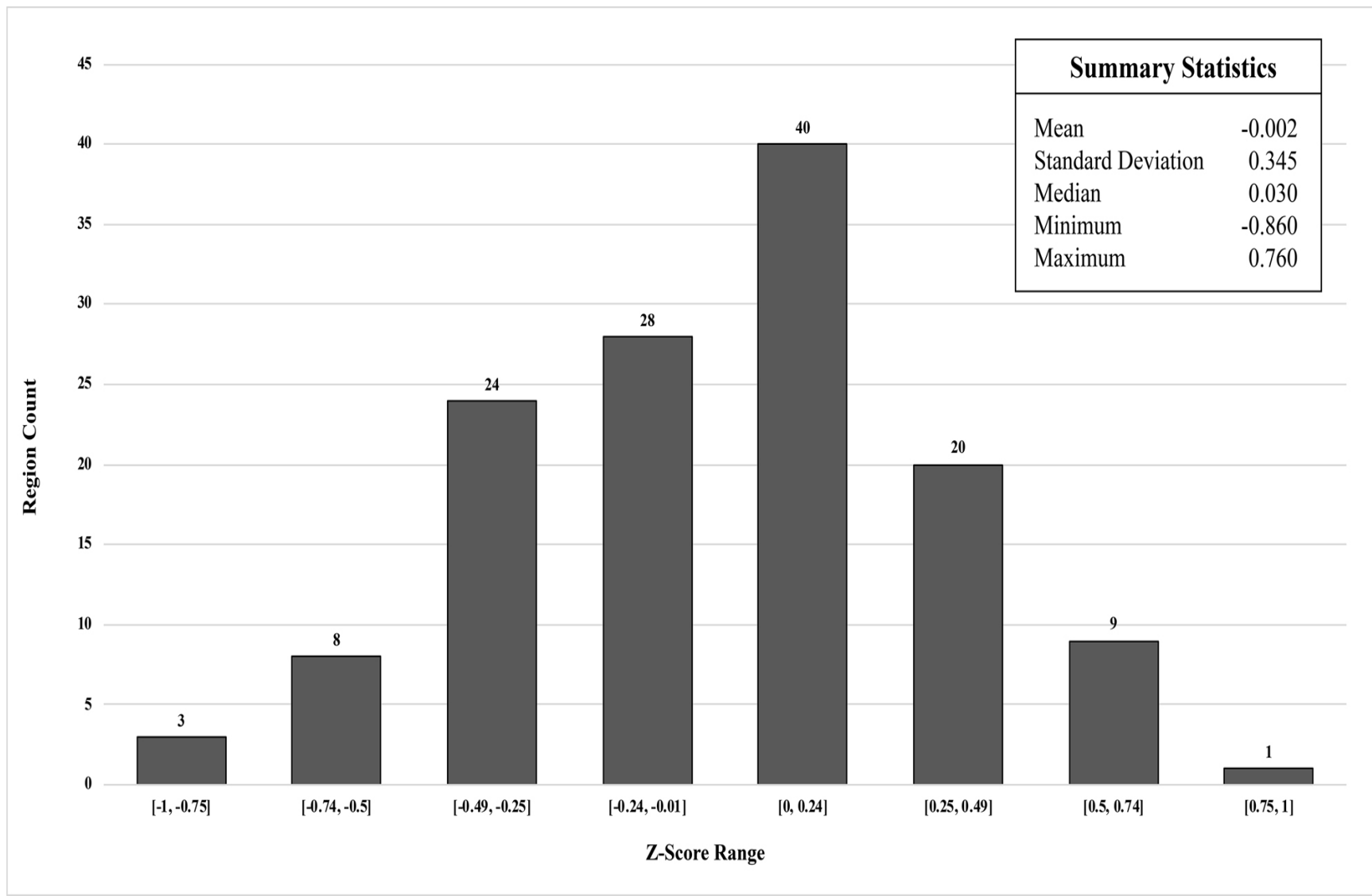

Figure 3. Histogram of region mean z-scores and their summary statistics from comparison of population template to MIMneuro ${ }^{\circledR}$ controls

Table 4. Summary statistics for clusters identified in population template using MIMneuro ${ }^{\circledR}$ voxel analysis. Parameters were a $P$-value of 1 minimum volume of $0 \mathrm{~mL}$, and minimum z-score of 1.65

\begin{tabular}{llllllllll}
\hline Statistic & Cluster $\mathbf{1}$ & Cluster $\mathbf{2}$ & Cluster $\mathbf{3}$ & Cluster $\mathbf{4}$ & Cluster $\mathbf{5}$ & Cluster $\mathbf{6}$ & Cluster $\mathbf{7}$ & Cluster 8 & Cluster $\mathbf{9}$ \\
\hline Mean & 1.90 & 1.87 & -1.85 & -1.66 & -1.67 & -1.67 & -1.74 & -1.71 & 1.66 \\
Standard deviation & 0.21 & 0.14 & 0.16 & 0.01 & 0.01 & 0.01 & 0.04 & 0.03 & 0.00 \\
Median & 1.84 & 1.85 & -1.81 & -1.66 & -1.67 & -1.67 & -1.72 & -1.71 & 1.66 \\
Minimum & 1.65 & 1.65 & -2.22 & -1.67 & -1.68 & -1.69 & -1.78 & -1.74 & 1.66 \\
Maximum & 2.65 & 2.19 & -1.65 & -1.65 & -1.66 & -1.65 & -1.69 & -1.67 & 1.66 \\
Volume (mL) & 11.53 & 2.62 & 1.67 & 0.10 & 0.08 & 0.05 & 0.04 & 0.02 & 0.02 \\
$P$-value & 0.93 & 0.99 & 0.99 & 1.00 & 1.00 & 1.00 & 1.00 & 1.00 & 1.00 \\
\hline
\end{tabular}




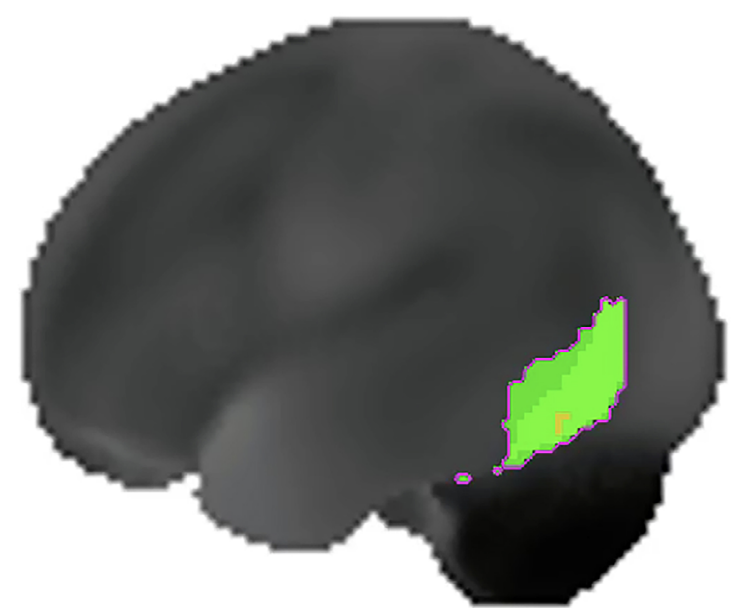

Figure 4. The largest cluster $(P=0.93)$ identified in the population template using MIMneuro ${ }^{\circledR}$ cluster analysis tools spanned portions of the left temporal and occipital lobes. This image depicts the left hemisphere surface projection of the population template with the cluster highlighted in green

\section{ROI analysis against template and normative database}

Probability plots first confirmed that the data and their differences were approximately normally distributed (Figures S1-3). Scaled and unscaled agreement indices are summarized for each region in Table 5. The first scaled metric calculated was the CCC, a dimensionless quantity that can vary from - 1 to 1 , similar to Pearson's correlation coefficient. When interpreted the same way, the CCCs generated from our data indicated poor to moderate agreement between the softwares, with CCC CIs ranging from 0.339 to 0.888 [34]. The ICC $(3,1)$ is related to the CCC but instead evaluates consistency between softwares such that their measurements are allowed to differ with some amount of error. In our data, the ICC CIs ranged from 0.555 to 0.905 , indicating moderate to good consistency between the softwares [44]. Both the CCC and ICC are dimensionless metrics and were therefore not compared to the noninferiority margin.

Table 5. Scaled (CCC, ICC) and unscaled (MSD, TDI) agreement metrics and 95\% Cls calculated between CereMetrix ${ }^{\circledR}$ data and MIMneuro ${ }^{\circledR}$ data

\begin{tabular}{|c|c|c|c|c|c|}
\hline Region & & $\operatorname{CCC}[95 \% \mathrm{Cl}]$ & $\operatorname{ICC}(3,1)[95 \% \mathrm{CI}]$ & MSD [95\% Cl] & $\mathrm{TDI}_{95 \%}[95 \% \mathrm{Cl}]$ \\
\hline \multirow[t]{4}{*}{ Frontal lobe } & $\mathrm{L}$ & 0.730 & 0.790 & 0.064 & 0.498 \\
\hline & & {$[0.607,0.819]$} & {$[0.661,0.874]$} & {$[0.044,0.094]$} & {$[0.409,0.604]$} \\
\hline & $\mathrm{R}$ & 0.608 & 0.758 & 0.097 & 0.613 \\
\hline & & {$[0.464,0.720]$} & {$[0.613,0.853]$} & {$[0.070,0.137]$} & {$[0.503,0.743]$} \\
\hline \multirow[t]{4}{*}{ Parietal lobe } & $\mathrm{L}$ & 0.820 & 0.841 & 0.067 & 0.508 \\
\hline & & {$[0.718,0.888]$} & {$[0.738,0.905]$} & {$[0.046,0.099]$} & {$[0.418,0.617]$} \\
\hline & $\mathrm{R}$ & 0.795 & 0.818 & 0.076 & 0.542 \\
\hline & & {$[0.679,0.873]$} & {$[0.703,0.891]$} & {$[0.052,0.113]$} & {$[0.446,0.659]$} \\
\hline \multirow[t]{4}{*}{ Temporal lobe } & $\mathrm{L}$ & 0.500 & 0.718 & 0.168 & 0.805 \\
\hline & & {$[0.339,0.633]$} & {$[0.555,0.828]$} & {$[0.123,0.231]$} & {$[0.663,0.970]$} \\
\hline & $\mathrm{R}$ & 0.760 & 0.767 & 0.068 & 0.512 \\
\hline & & {$[0.643,0.843]$} & {$[0.626,0.859]$} & {$[0.046,0.101]$} & {$[0.421,0.623]$} \\
\hline \multirow[t]{4}{*}{ Internal structures } & $L$ & 0.806 & 0.812 & 0.110 & 0.652 \\
\hline & & {$[0.718,0.868]$} & {$[0.694,0.888]$} & {$[0.075,0.163]$} & {$[0.536,0.792]$} \\
\hline & $\mathrm{R}$ & 0.814 & 0.821 & 0.086 & 0.574 \\
\hline & & {$[0.729,0.875]$} & {$[0.708,0.893]$} & {$[0.058,0.127]$} & {$[0.472,0.697]$} \\
\hline
\end{tabular}

MSDs are the expected squared differences between the softwares' measurements. Satisfactory agreement is reached when MSDs are less than or equal to the square of the noninferiority margin, or 2.72 [37]. The 95\% CIs for MSDs in our data were much less than that, ranging from 0.044 to 0.231 . 
TDI is the absolute difference in measurements that achieves a desired coverage probability. In our data, TDI CIs computed to achieve $95 \%$ coverage probability range from 0.409 to 0.970 z-score. Satisfactory agreement is achieved when the TDI is less than the noninferiority margin, which was true here.

While unscaled indices (MSD, TDI) suggested CereMetrix ${ }^{\circledR}$ results were substantially noninferior to MIMneuro ${ }^{\circledR}$, the scaled indices (CCC, ICC) did not support full agreement. Lines of equality showed this clearly; paired measurements between the softwares trend with the equality line but are scattered around it (Figures 5 and 6). CereMetrix ${ }^{\circledR}$ results do not exactly equal MIMneuro ${ }^{\circledR}$ results.
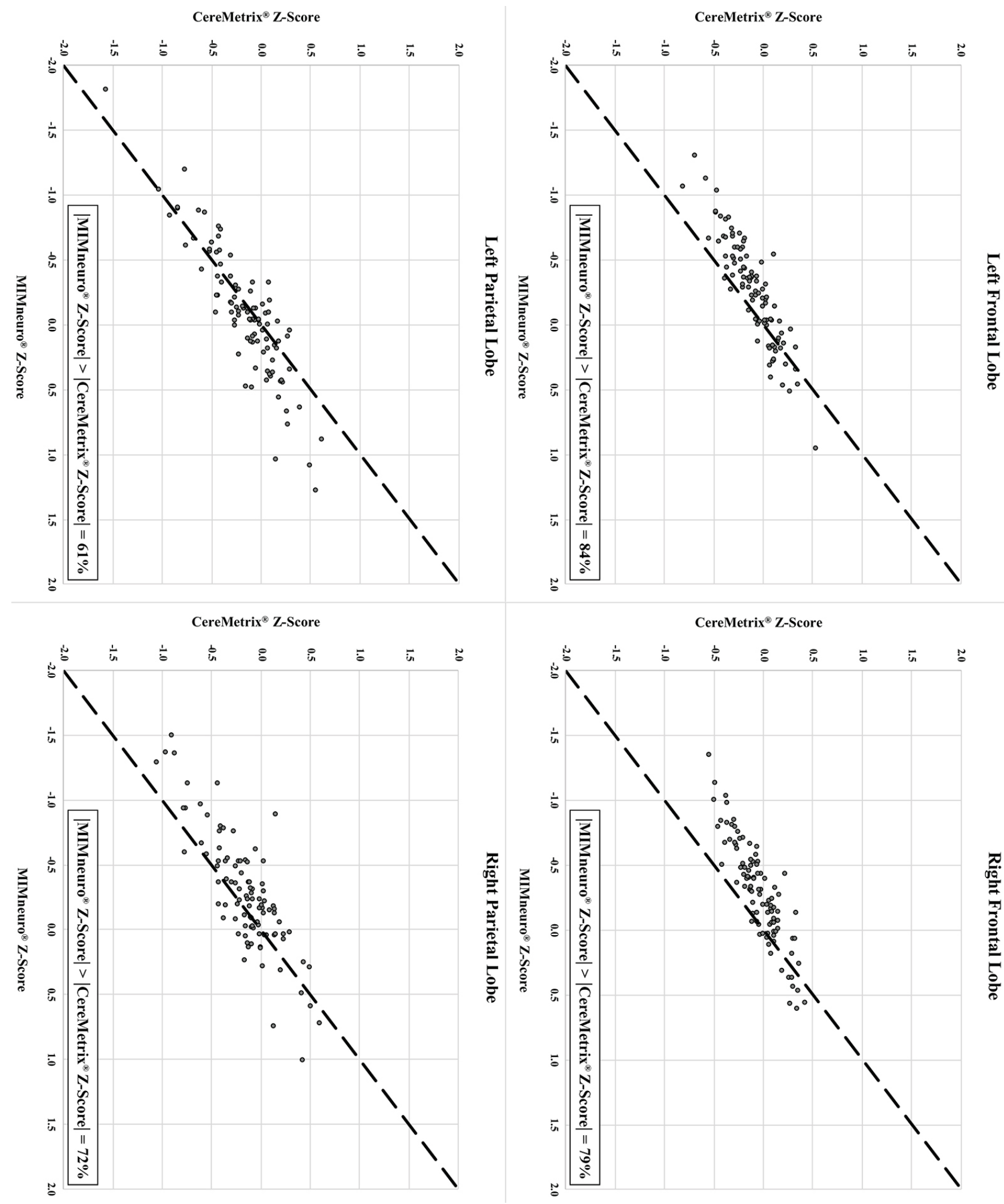

Figure 5. Frontal and parietal lobe equality plots between CereMetrix ${ }^{\circledast}$ and MIMneuro ${ }^{\circledR}$ data showing their scatter around the line of equality. The proportion of measurements where MIMneuro ${ }^{\circledR}$ 's $z$-score magnitude was greater than the corresponding CereMetrix $^{\circledR} \mathrm{z}$-score magnitude is also given 

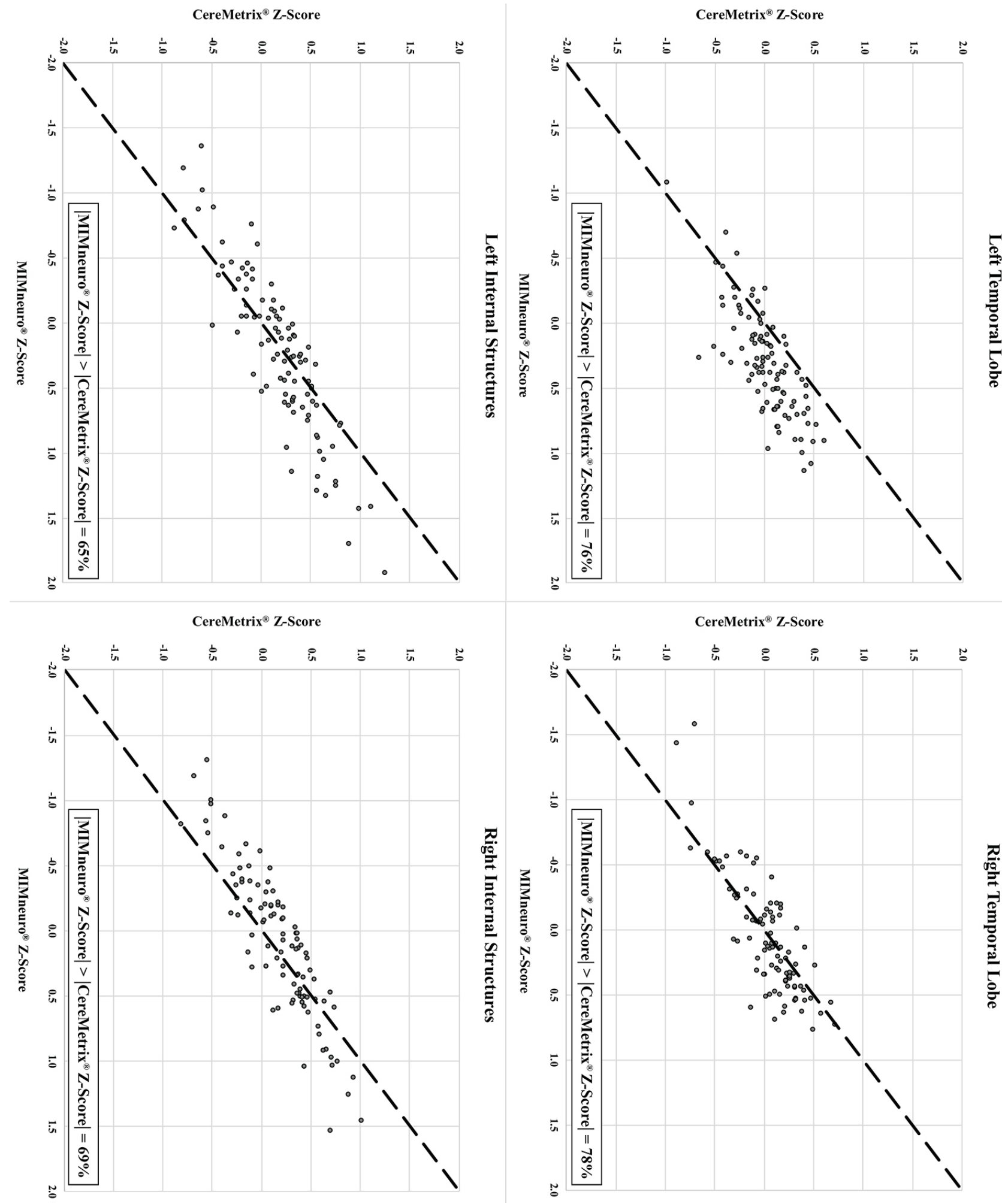

Figure 6. Temporal lobe and internal structures equality plots between CereMetrix ${ }^{\circledR}$ and MIMneuro $^{\circledR}$ data showing their scatter around the line of equality. The proportion of measurements where MIMneuro ${ }^{\circledR}$ 's Z-score magnitude was greater than the corresponding CereMetrix ${ }^{\circledR}$ z-score magnitude is also given

These differences between the softwares were evaluated with Bland-Altman plots and LOAs. The traditional horizontal LOA plots revealed proportional bias and slight heteroscedasticity that could not be remedied by recommended methods like ratios, percent differences, or log transformations (Figure 7). Therefore, simple linear regression was used to find the line of best fit and new LOAs (Figure 8) [39, 40, $45,46]$. LOAs define the range where most differences between these softwares are expected to lie. This was true for our data, as $97-99 \%$ fell between the confidence bounds of these LOAs. 



Figure 7. Bland-Altman plots with horizontal LOAs and Cls for each region. While much of the data is contained within the LOAs, proportional bias is evident 

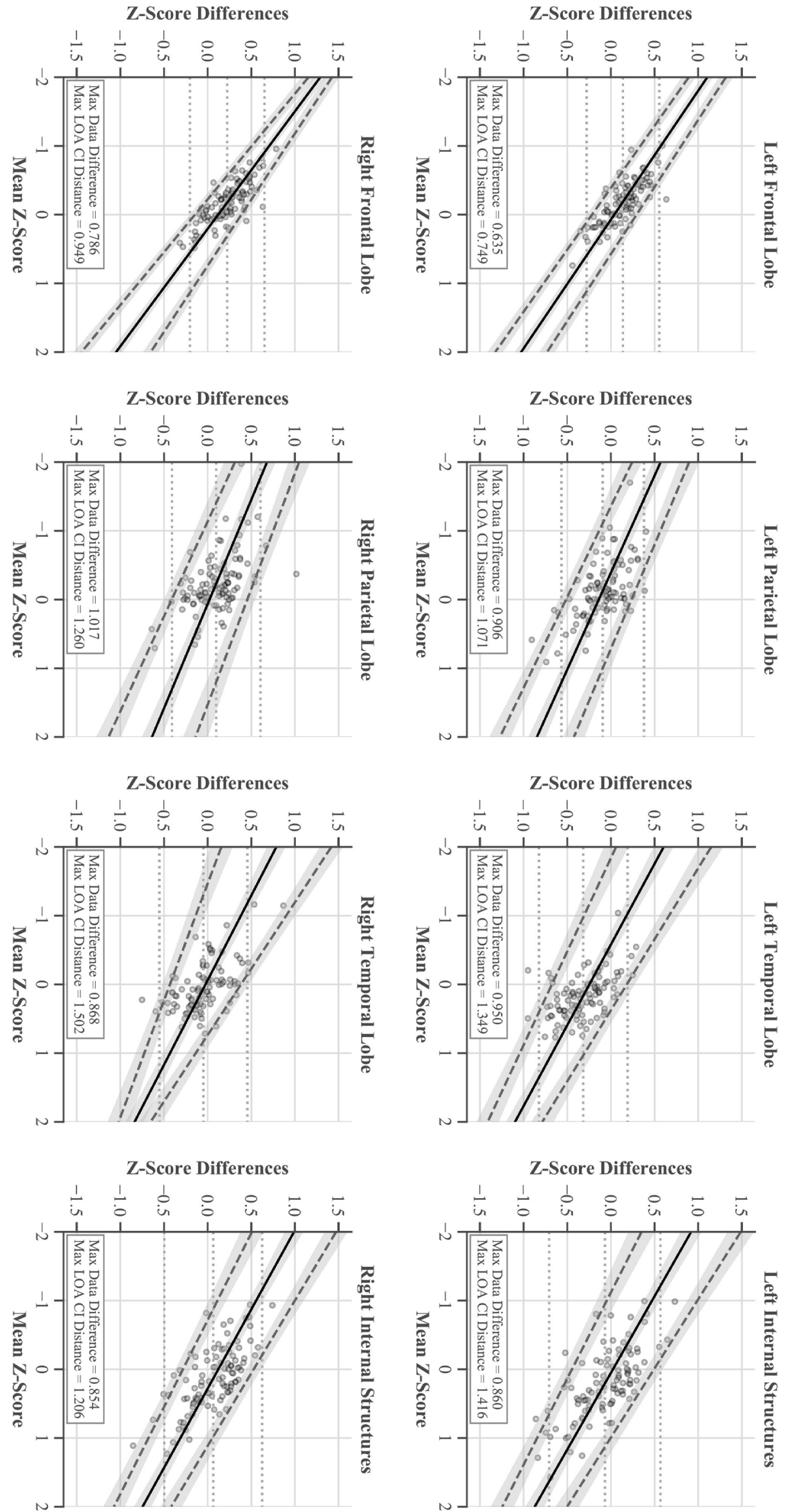

Figure 8. Bland-Altman plots with linear LOAs and Cls for each region accounting for proportional bias present in the data. Original mean and horizontal LOAs are represented by light gray dashed lines 
The largest single difference between the softwares was $1.017 \mathrm{z}$-score and occurred in the right parietal lobe. The largest systemic difference was in the left temporal lobe, with its average differences amounting to -0.32 z-score while the others ranged from -0.09 to 0.22 (Figure 7).

For each ROI, proportional bias manifested as a negative relationship between software differences and the mean of their values. This relationship was most pronounced in the frontal lobes and least in the parietal lobes. Since all differences were calculated by subtracting MIMneuro ${ }^{\circledR}{ }^{\circledR} \mathrm{s}$ value from CereMetrix ${ }^{\circledR}$ 's, the negative slope was caused by MIMneuro ${ }^{\circledR}$ 's z-score magnitude consistently exceeding CereMetrix ${ }^{\circledR} \mathrm{s}$ in $61-84 \%$ of each region's measurements (Figures 5 and 6 ).

LOAs were narrowest in the frontal lobes and widest in the right temporal lobe. This region also contained the most noticeable heteroscedasticity, with variability decreasing for positive means and increasing for negative means. No individual measurement differences exceeded \pm 1.65 z-score and none of the LOA CIs extended beyond those boundaries when the mean z-score was between -2 and 2 . Thus we are $95 \%$ confident that the difference in future measurements would also be within $\pm 1.65 \mathrm{z}$-score.

\section{Visual reads after template comparison}

Finally, the single reader fully agreed with the impressions documented by the original physicians in 80 of the 100 cases in the cognitively impaired cohort. Krippendorff's alpha was 0.72 with a $95 \%$ CI of 0.61 to 0.82 . In 10 cases, the reader agreed with a portion of the impressions but not the final conclusions. The remaining 10 cases were completely discordant. Examples from the cohort analyzed in CereMetrix ${ }^{\circledR}$ presenting established perfusion patterns of AD, FTD, and TBI are given in Figures 9-11.

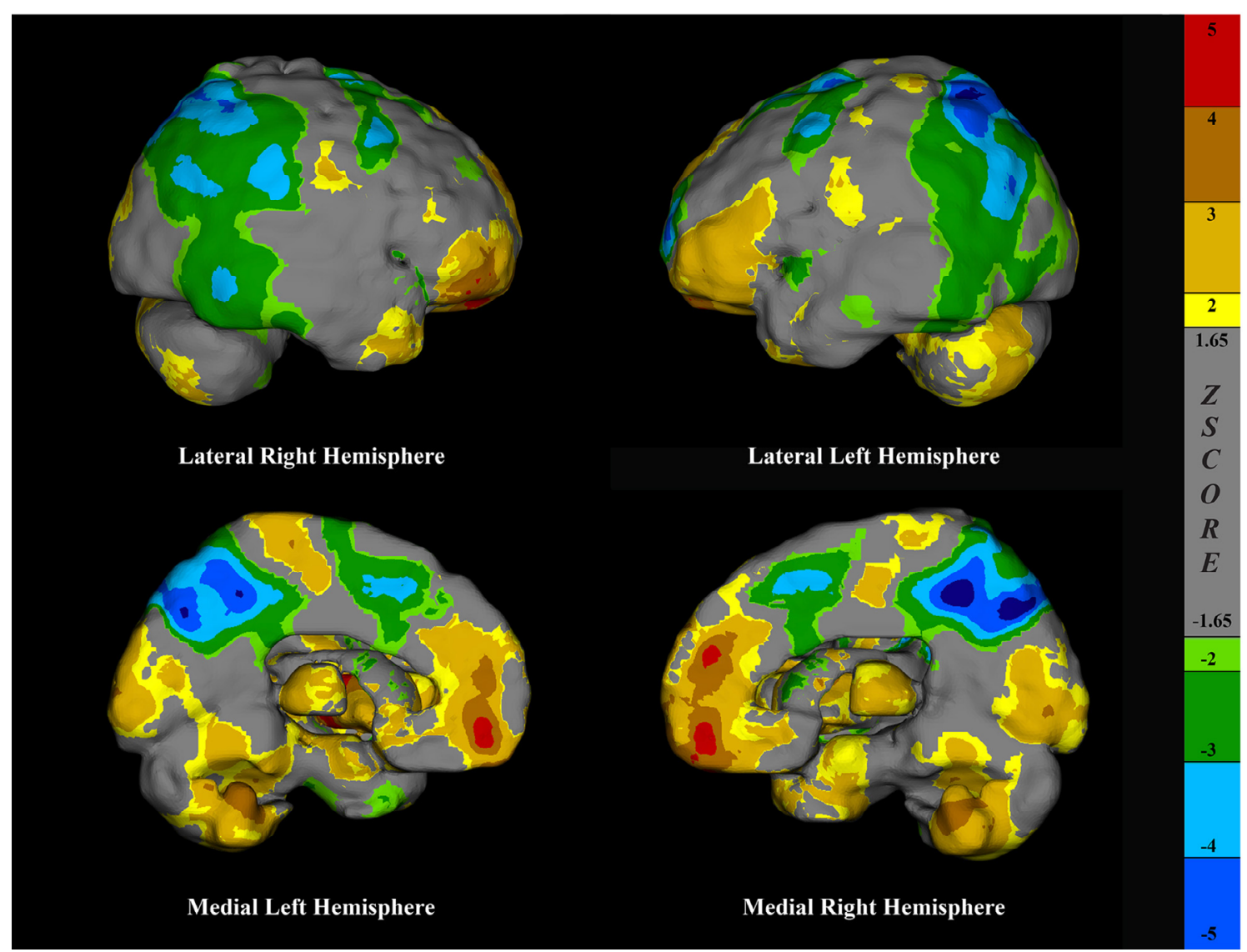

Figure 9. Surface projections of a brain SPECT scan rendered in CereMetrix ${ }^{\circledR}$ demonstrating temporal parietal hypoperfusion in a person with suspected $A D$ 


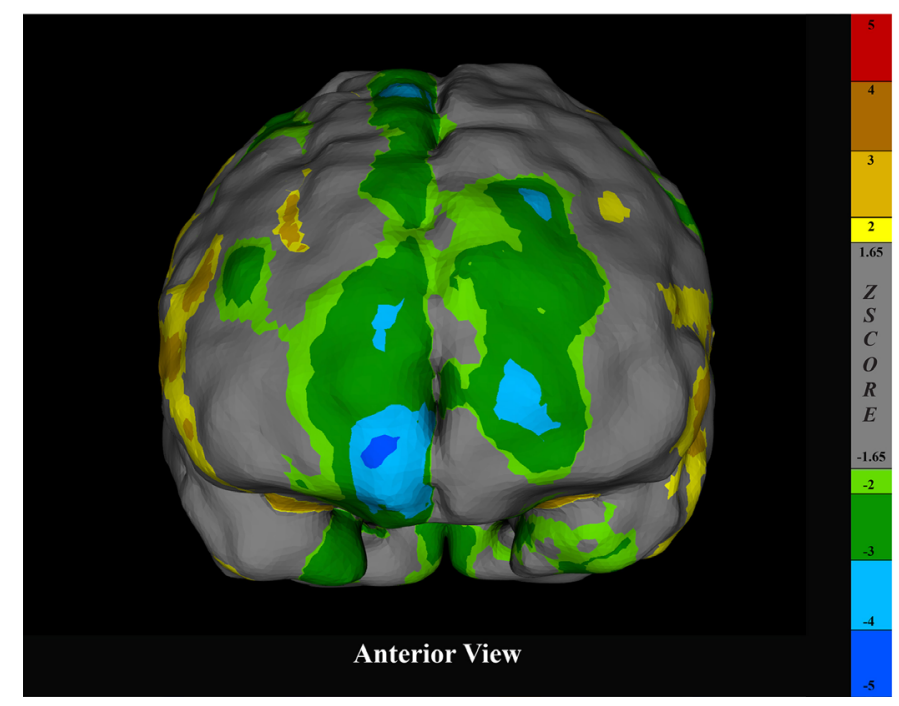

Figure 10. Surface projection of a brain SPECT scan rendered in CereMetrix ${ }^{\circledR}$ demonstrating frontal hypoperfusion in a person with suspected FTD

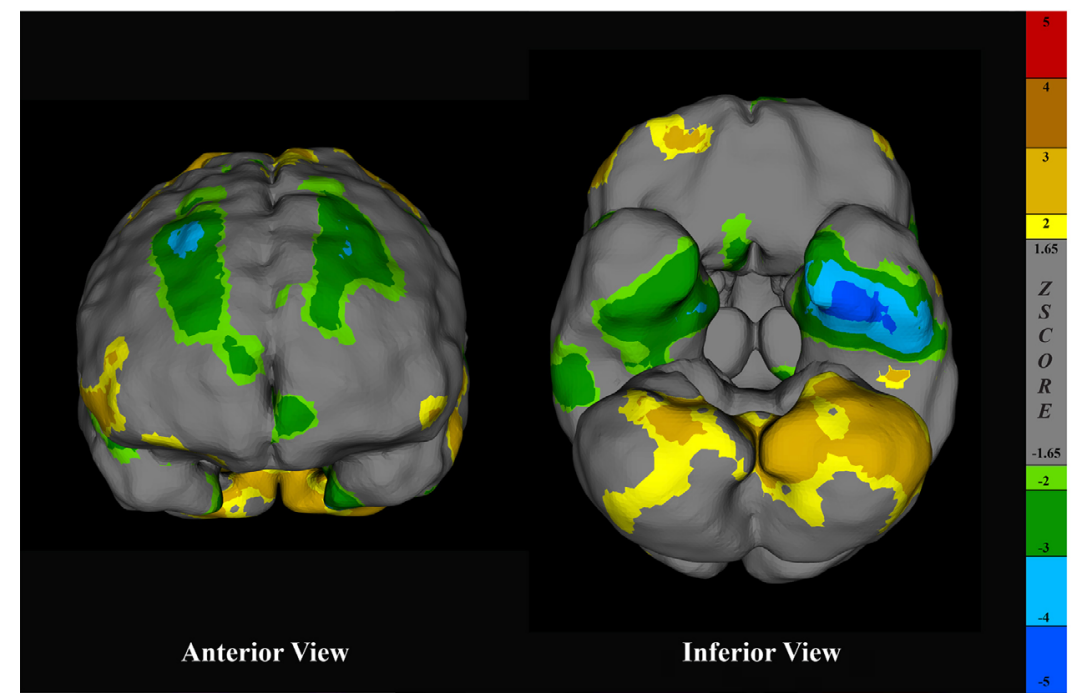

Figure 11. Surface projections of a brain SPECT scan rendered in CereMetrix ${ }^{\circledR}$ demonstrating asymmetric frontal and temporal hypoperfusion in a person with suspected TBI

\section{Discussion}

Quantitative metrics for brain SPECT analysis are dependent on normative datasets but building them is challenging and expensive. For such investment, the number of participants in commercial normative datasets is frequently small [13,19-21], limiting age ranges and subsequent comparisons to single subjects [47]. This study created an alternative to traditional normative datasets using a large clinical collection of abnormal scans and established noninferiority to the controls contained in a commercial product.

Various normative datasets have characterized cerebral perfusion across SPECT tracers $[25,48,49]$, age or sex groups [50-54], spatial normalization templates [55, 56], and processing or equipment [57-59], yet no work to our knowledge has ever compared control databases of commercial products to each other. Controls are essential to any study investigating abnormal perfusion patterns, but the diseased cohort typically homogenous by design. No other group has attempted to substantiate noninferiority between a heterogeneous cohort of abnormal brain SPECT scans and a set of controls.

Direct quantitative comparison demonstrated that the population template was noninferior to MIMneuro ${ }^{\circledR \prime}$ 's normative dataset. Despite this, CereMetrix ${ }^{\circledR}$ and MIMneuro ${ }^{\circledR}$ softwares do not employ identical processing methods when comparing individual scans against their respective datasets. Investigation 
of their quantitative results on individual subjects was warranted to ensure noninferiority was sustained following integration of the template into the CereMetrix ${ }^{\circledR}$ pipeline.

Metrics generated from both softwares' region-based data gave mixed results. CCCs and ICCs indicated poor to moderate agreement yet moderate to good consistency between the software measurements, but these indices are difficult to interpret from a clinical perspective because they are dimensionless. MSDs and TDIs were calculated for this purpose and they suggested satisfactory agreement and noninferiority.

The equality and Bland-Altman plots revealed why the results from the scaled and unscaled indices were seemingly inconsistent. While the CereMetrix ${ }^{\circledR}$ and MIMneuro ${ }^{\circledR}$ measurements trend with each other, they were not equal and proportional bias existed between them. This bias was caused in part by the magnitude of MIMneuro ${ }^{\circledR}$ 's measurements often exceeding those of CereMetrix ${ }^{\circledR}$. However, no individual differences or LOAs crossed the noninferiority margin. From a clinical perspective, this is satisfactory agreement and CereMetrix ${ }^{\circledR}$ quantification using the population template is noninferior to MIMneuro ${ }^{\circledR}$ 's quantification using their normative dataset.

Nonetheless, the bias observed in the Bland-Altman plots cannot be ignored. The mean of the measurements within our data did not exceed \pm 2 z-score but it is clear that the LOAs may eventually cross the noninferiority margin as z-score magnitude grows. We find this unlikely for the broad anatomical regions analyzed here, but if the relationship were maintained for smaller structures, differences in z-score magnitude may be unsatisfactory. Our data cannot make any conclusions on this possibility. In practice, this bias means that quantitative results from MIMneuro ${ }^{\circledR}$ could be more extreme in magnitude and yield less specific perfusion pattern results than CereMetrix ${ }^{\circledR}$.

MIMneuro ${ }^{\circledR}$ 's default threshold for normality is $\pm 1.65 \mathrm{z}$-score though some softwares use $\pm 2 \mathrm{z}$-score to increase the specificity of their results [60]. Within these bounds, the differences between the softwares are acceptable. However, from a clinical perspective, once an area of the brain crosses those thresholds, is it the z-score magnitude or the spatial pattern of abnormalities that dictates the diagnosis? If the former, the risk of software differences interfering with clinical decision making is legitimate. If the latter, increasing differences between the softwares at more extreme z-scores become immaterial.

Our work did not investigate how perfusion quantification is ultimately translated into diagnostic impression, but we did need to ensure consistency of clinical reads between the softwares. The secondary reads performed on the cognitively impaired cohort demonstrated substantial concordance between the single reader using the population template within CereMetrix ${ }^{\circledR}$ and the original impressions produced by six other readers. While not perfect, the observed Krippendorff's alpha and percent agreement were consistent with or exceeded those previously recorded [61-65].

Direct comparison of our results to these dated works is complicated by key differences in SPECT scan presentation. All performed strictly two-dimensional visual analyses, at least two used films instead of computer displays for scan review [61, 62], and two reported using grayscale instead of applying color tables [61, 62]. These methodological choices now conflict with ACR guidelines for brain SPECT, which explicitly recommend computer display for three-dimensional viewing, color table adjustment, and quantitative analysis [5]. Additionally, Hellman's and Pasquier's work evaluated agreement in scan appearance or ROI severity ratings instead of the resulting diagnosis. Our study, like Stockbridge, Doran, and Barnes, measured agreement in diagnostic interpretation. Only Barnes introduced software assistance from Statistical Parametric Mapping, reporting a kappa of 0.5 between the readers. We are unaware of any recent work that has evaluated interobserver variability of diagnostic impression in Tc99m-HMPAO brain SPECT using quantitative software.

Our study achieved full concordance in $80 \%$ of the cases, partial concordance in $10 \%$, and full discordance in $10 \%$. While our statistics were conservative in considering partial concordance as disagreement, the data were more nuanced. In seven of the partial concordance cases, one reader proposed a mixed etiology of two conditions while the other selected one of those conditions exclusively. In the other three cases, one reader chose a primary condition but noted a second possibility while the other considered this second condition 
to be the primary. This potentially highlights differences in reader interpretation rather than differences in perfusion pattern.

Such interobserver variability is not uncommon, particularly in the context of clinical cognitive impairment data [64]. Gold standard diagnoses for this patient group can only be determined postmortem [66], challenging all dementia research. Despite this, our data's consistency with prior work indicates that established perfusion patterns for conditions like AD [67, 68], FTD [69, 70], and TBI $[71,72]$ were in fact observable after comparison to the population template.

Our work was limited by the inability to perform raw voxel comparisons between the population template and any other dataset of controls. Instead, direct comparisons were limited to the tools available through MIMneuro ${ }^{\circledR}$ 's interface. A number of methodological differences may have also broadly influenced our results, including acquisition and reconstruction equipment and parameters, processing algorithms, reference templates and atlases, and anatomical region parcellations. Notwithstanding these challenges, our work provides a unique, though seemingly incongruous, alternative to normative datasets.

It is possible that these results would change if a subset of the scans used for the template were selected or if more were added, particularly if the resulting dataset was biased toward a specific condition. Our approach depends on data heterogeneity. We presumed heterogeneity given the clinical nature of these scans and the array of conditions within it, but the diagnostic impression labels assigned to them do not guarantee heterogeneity across each image's voxels. How to define and measure sufficient heterogeneity to model normal perfusion is an open question. Our only solution, for now, is to validate each newly generated template independently.

This is why future work will require similar validation of the population template when segmented by age or sex. It is well documented that perfusion patterns change over the course of our lifetimes [50-54] so the ability to compare individual patients to subjects within their age range is imperative. We will be challenged to find suitable comparison datasets that are representative of normal perfusion in limited age ranges, particularly for children and young adults. For example, though MIMneuro ${ }^{\circledR}$ contains one of the largest normative datasets, their young adult population only has three controls and may not constitute an ideal reference.

\section{Conclusion}

Quantifying image-derived metrics is of increasing interest across medical specialties but doing so for brain SPECT analysis is dependent on normative datasets that are challenging and expensive to produce. Instead of controls, we used clinical scans to create an alternative, the population template. We investigated its utility for perfusion measurement in three ways. First, direct analysis of the template against a normative dataset demonstrated it was noninferior both at the region and voxel level. Second, using a cohort of cognitively impaired individuals, z-score metrics derived from the template were also noninferior to those generated from the normative dataset. Finally, individual visual reads of the same cohort revealed that, following quantification using the template, the perfusion patterns observed were largely consistent with their original clinical read and established literature. Despite this study's limitations and recommendations for future work, we conclude that the population template is a viable alternative to normative datasets for quantifying brain SPECT.

\section{Abbreviations}

AAL: automatic anatomical labelling atlas

ACR: American College of Radiology

AD: Alzheimer's disease

CCC: concordance correlation coefficient

CI: confidence interval

FTD: frontotemporal dementia 
ICC $(3,1)$ : intraclass correlation coefficient (two way mixed, single measures, consistency)

LOA: limits of agreement

MI: mutual information

MNI: Montreal Neurological Institute

MSD: mean squared deviations

PET: positron emission tomography

ROI: regions of interest

SPECT: single photon emission computed tomography

TBI: traumatic brain injury

Tc99m-HMPAO: technetium-99m hexamethylpropyleneamine oxime

TDI: total deviation indices

\section{Supplementary materials}

The supplementary materials for this article are available at: https://www.explorationpub.com/uploads/ Article/file/100122_sup_1.pdf.

\section{Declarations}

\section{Acknowledgments}

We would like to thank CereHealth Corp. for their donation of patient data and support of this study, and individuals Shane Quint, Laurie Haynie, Michaela Olson, Logan Langholz, and Christine Salva for their contributions to this work.

\section{Author contributions}

LMQ contributed to the conception and design of the study. CAR also contributed to its design. With some assistance from acknowledged individuals, LMQ processed all clinical and imaging data for the template and the cognitively impaired cohort. LMQ generated the template and performed all testing and statistical analysis. CAR performed all visual reads on the cognitively impaired cohort. LMQ wrote the first draft of the manuscript. CAR wrote sections of the manuscript. All authors contributed to manuscript revision, read, and approved the submitted version.

\section{Conflicts of interest}

Lindsay Quandt is an employee of CereHealth Corporation, the parent company of CereScan and CereMetrix Corporations. Cyrus Raji is an independent contractor for CereHealth Corporation.

\section{Ethical approval}

This study was approved by the IntegReview Institutional Review Board, Certificate CHDB112019.

\section{Consent to participate}

The informed consent to participate in the study was obtained from all participants.

\section{Consent to publication}

Not applicable.

\section{Availability of data and materials}

The datasets generated and analyzed for this manuscript are not publicly available because they are proprietary resources of CereHealth Corporation. Requests for access should be directed to Lindsay M. Quandt (LQuandt@ceremetrix.io). 
Funding

Not applicable.

Copyright

(C) The Author(s) 2020.

\section{References}

1. Valotassiou V, Malamitsi J, Papatriantafyllou J, Dardiotis E, Tsougos I, Psimadas D, et al. SPECT and PET imaging in Alzheimer's disease. Ann Nucl Med. 2018;32:583-93.

2. Raji CA, Tarzwell R, Pavel D, Schneider H, Uszler M, Thornton J, et al. Clinical utility of SPECT neuroimaging in the diagnosis and treatment of traumatic brain injury: a systematic review. PLoS One. 2014;9:e91088.

3. Desikan R, Rafii M, Brewer J, Hess C. An expanded role for neuroimaging in the evaluation of memory impairment. Methodol Perspect Brain. 2013;34:2075-82.

4. Catafau AM. Brain SPECT in clinical practice. Part I: perfusion. J Nucl Med. 2001;42:259-71.

5. American College of Radiology Committee on Practice Parameters and Technical Standards. ACR-SPR practice parameter for the performance of single photon emission computed tomography (SPECT) brain perfusion imaging, including brain death examinations. 2016 [cited 2018 Sep 5]. Available from: https:// www.acr.org/-/media/ACR/Files/Practice-Parameters/brainperf-spect.pdf?la=en

6. Patterson JC, Early TS, Martin A, Walker MZ, Russell JM, Villanueva-Meyer H. SPECT image analysis using statistical parametric mapping: comparison of technetium-99m-HMPAO and technetium-99m-ECD. J Nucl Med. 1997;38:1721-5.

7. Bartenstein P, Minoshima S, Hirsch C, Buch K, Willoch F, Mösch D, et al. Quantitative assessment of cerebral blood flow in patients with Alzheimer's disease by SPECT. J Nucl Med. 1997;38:1095-101.

8. Ishii K, Kanda T, Uemura T, Miyamoto N, Yoshikawa T, Shimada K, et al. Computer-assisted diagnostic system for neurodegenerative dementia using brain SPECT and 3D-SSP. Eur J Nucl Med Mol Imaging. 2009;36:831-40.

9. Van Laere KJ, Warwick J, Versijpt J, Goethals I, Audenaert K, Van Heerden B, et al. Analysis of clinical brain SPECT data based on anatomic standardization and reference to normal data: an ROC-based comparison of visual, semiquantitative, and voxel-based methods. J Nucl Med. 2002;43:458-69.

10. Waragai M, Yamada T, Matsuda H. Evaluation of brain perfusion SPECT using an easy Z-score imaging system (eZIS) as an adjunct to early-diagnosis of neurodegenerative diseases. J Neurol Sci. 2007;260:57-64.

11. Imabayashi E, Matsuda H, Asada T, Ohnishi T, Sakamoto S, Nakano S, et al. Superiority of 3-dimensional stereotactic surface projection analysis over visual inspection in discrimination of patients with very early Alzheimer's disease from controls using brain perfusion SPECT. J Nucl Med. 2004;45:1450-7.

12. Radau PE, Slomka PJ, Julin P, Svensson L, Wahlund LO. Evaluation of linear registration algorithms for brain SPECT and the errors due to hypoperfusion lesions. Med Phys. 2001;28:1660-8.

13. MIM Software Inc. MIMneuro ${ }^{\circledR}$. Version 6.7.12 [software]. 2018 [cited 2018 Sep 5]. Available from: https://www.mimsoftware.com/nuclear_medicine/mim_neuro

14. Kessler LG, Barnhart HX, Buckler AJ, Choudhury KR, Kondratovich MV, Toledano A, et al. The emerging science of quantitative imaging biomarkers terminology and definitions for scientific studies and regulatory submissions. Stat Methods Med Res. 2015;24:9-26.

15. Jha AK, Caffo B, Frey EC. A no-gold-standard technique for objective assessment of quantitative nuclearmedicine imaging methods. Phys Med Biol. 2016;61:2780-800.

16. Jha AK, Song N, Caffo B, Frey EC. Objective evaluation of reconstruction methods for quantitative SPECT imaging in the absence of ground truth. Proc SPIE Int Soc Opt Eng. 2015;9416:94161K. 
17. Smith ND, Holmes RB, Soleimani M, Evans MJ, Cade SC, Mitchell CN. Towards adapting a normal patient database for SPECT brain perfusion imaging. Inverse Probl. 2012;28:065001.

18. Radiation and your patient: a guide for medical practitioners. Ann ICRP. 2001;31:5-31.

19. Barnden LR, Behin-Ain S, Kwiatek R, Casse R, Yelland L. Age related preservation and loss in optimized brain SPECT. Nucl Med Commun. 2005;26:497-503.

20. Segami Corporation. Oasis. Version 1.9.4.9 [software]. 2018 [cited 2018 Sep 5]. Available from: https:// segamicorp.com

21. HERMES Medical Solutions. BRASS ${ }^{\mathrm{TM}}$. Version 3.6 [software]. 2013 [cited 2018 Sep 5]. Available from: https://www.hermesmedical.com

22. Yeo JM, Lim X, Khan Z, Pal S. Systematic review of the diagnostic utility of SPECT imaging in dementia. Eur Arch Psychiatry Clin Neurosci. 2013;263:539-52.

23. Swan A, Waddell B, Holloway G, Bak T, Colville S, Khan Z, et al. The diagnostic utility of 99mTc-HMPAO SPECT imaging: a retrospective case series from a tertiary referral early-onset cognitive disorders clinic. Dement Geriatr Cogn Disord. 2015;39:186-93.

24. Prosser AMJ, Tossici-Bolt L, Kipps CM. The impact of regional 99mTc- HMPAO single-photon-emission computed tomography (SPECT) imaging on clinician diagnostic confidence in a mixed cognitive impairment sample. Clin Radiol. 2020;75:714.e7-714.e14.

25. Koyama M, Kawashima R, Ito H, Ono S, Sato K, Goto R, et al. SPECT imaging of normal subjects with technetium-99m-HMPAO and technetium-99m-ECD. J Nucl Med. 1997;38:587-92.

26. Chang LT. A method for attenuation correction in radionuclide computed tomography. IEEE Trans Nucl Sci. 1978;25:638-43.

27. Matsumoto N, Berman DS, Kavanagh PB, Gerlach J, Hayes SW, Lewin HC, et al. Quantitative assessment of motion artifacts and validation of a new motion- correction program for myocardial perfusion SPECT. J Nucl Med. 2001;42:687-94.

28. Otsu N. A threshold selection method from gray-level histogram. IEEE Trans Syst Man Cybern. 1979;9:62-6.

29. Holmes CJ, Hoge R, Collins L, Woods R, Toga AW, Evans AC. Enhancement of MR images using registration for signal averaging. J Comput Assist Tomogr. 1998;22:324-33.

30. Arndt S, Cizadlo T, O'Leary D, Gold S, Andreasen NC. Normalizing counts and cerebral blood flow intensity in functional imaging studies of the human brain. Neuroimage. 1996;3:175-84.

31. Obuchowski NA, Reeves AP, Huang EP, Wang XF, Buckler AJ, Kim HJ, et al; Algorithm Comparison Working Group. Quantitative imaging biomarkers: a review of statistical methods for computer algorithm comparisons. Stat Methods Med Res. 2015;24:68-106.

32. Piper JW, Corp M. Quantitative comparison of spatial normalization algorithms for 3D PET brain scans. J Nucl Med. 2007;48 Suppl 2:403.

33. Bonferroni CE. Teoria statistica delle classi e calcolo delle probabilita. Pubbl del R Ist Super di Sci Econ e Commer di Firenze. 1936;8:3-62.

34. Devore JL. Probability and Statistics for Engineering and the Sciences. 8th ed. Boston (MA): Cengage Learning; 2012.

35. Rolls ET, Joliot M, Tzourio-Mazoyer N. Implementation of a new parcellation of the orbitofrontal cortex in the automated anatomical labeling atlas. Neuroimage. 2015;122:1-5.

36. Lin L, Hedayat AS, Sinha B, Yang M. Statistical methods in assessing agreement: models, issues, and tools. J Am Stat Assoc. 2002;97:257-70.

37. Barnhart HX, Haber MJ, Lin LI. An overview on assessing agreement with continuous measurements. J Biopharm Stat. 2007;17:529-69. 
38. Lin L. Total deviation index for measuring individual agreement with applications in laboratory performance and bioequivalence. Stat Med. 2000;19:255-70.

39. Choudhary PK, Nagaraja HN. Measuring agreement in method comparison studies-a review. In: Balakrishnan N, Nagaraja HN, Kannan N, editors. Advances in ranking and selection, multiple comparisons, and reliability. Boston (MA): Birkhäuser; 2005. pp. 215-44.

40. Bland JM, Altman DG. Measuring agreement in method comparison studies. Stat Methods Med Res. 1999;8:135-60.

41. Krippendorff KH. Content analysis: an introduction to its methodology. 2nd ed. Thousand Oaks (CA): Sage Publications, Inc.; 2004.

42. Hayes AF, Krippendorff K. Answering the call for a standard reliability measure for coding data. Commun Methods Meas. 2007;1:77-89.

43. Landis JR, Koch GG. The measurement of observer agreement for categorical data. 1977;33:159-74.

44. Koo TK, Li MY. A guideline of selecting and reporting intraclass correlation coefficients for reliability research. J Chiropr Med. 2016;15:155-63.

45. Chhapola V, Kanwal SK, Brar R. Reporting standards for Bland-Altman agreement analysis in laboratory research: a cross-sectional survey of current practice. Ann Clin Biochem. 2015;52:382-6.

46. Ludbrook J. Confidence in Altman-Bland plots: a critical review of the method of differences. Clin Exp Pharmacol Physiol. 2010;37:143-9.

47. Lobaugh NJ, Caldwell CB, Black SE, Leibovitch FS, Swartz RH. Three brain SPECT region-of-interest templates in elderly people: normative values, hemispheric asymmetries, and a comparison of singleand multihead cameras. J Nucl Med. 2000;41:45-56.

48. Ito H, Inoue K, Goto R, Kinomura S, Taki Y, Okada K, et al. Database of normal human cerebral blood flow measured by SPECT: I. Comparison between I-123-IMP, Tc-99m-HMPAO, and Tc-99m-ECD as referred with 0-15 labeled water PET and voxel-based morphometry. Ann Nucl Med.2006;20:131-8.

49. Brinkmann BH, Jones DT, Stead M, Kazemi N, O'Brien TJ, So EL, et al. Statistical parametric mapping demonstrates asymmetric uptake with Tc-99m ECD and Tc-99m HMPAO SPECT in normal brain. J Cereb Blood Flow Metab. 2012;32:190-8.

50. Yamamoto H, Arimura S, Nakanishi A, Shimo Y, Motoi Y, Ishiguro K, et al. Age-related effects and gender differences in Japanese healthy controls for [123I] FP-CIT SPECT. Ann Nucl Med. 2017;31:407-12.

51. Van Laere KJ, Dierckx RA. Brain perfusion SPECT: age- and sex-related effects correlated with voxelbased morphometric findings in healthy adults. Radiology. 2001;221:810-7.

52. Goto R, Kawashima R, Ito H, Koyama M, Sato K, Ono S, et al. A comparison of Tc-99m HMPAO brain SPECT images of young and aged normal individuals. Ann Nucl Med. 1998;12:333-9.

53. Pagani M, Salmaso D, Jonsson C, Hatherly R, Jacobsson H, Larsson SA, et al. Regional cerebral blood flow as assessed by principal component analysis and 99mTc-HMPAO SPET in healthy subjects at rest: normal distribution and effect of age and gender. Eur J Nucl Med Mol Imaging. 2002;29:67-75.

54. Schiepers C, Verbruggen A, Casaer P, Roo M De. Normal brain perfusion pattern of technetium-99methylcysteinate dimer in children. J Nucl Med. 1997;38:1115-20.

55. Morbelli S, Rodriguez G, Mignone A, Altrinetti V, Brugnolo A, Piccardo A, et al. The need of appropriate brain SPECT templates for SPM comparisons. Q J Nucl Med Mol Imaging. 2008;52:89-98.

56. Yang BH, Wang SJ, Chou YH, Su TP, Chen JC. The effect of templates on 99mTc-ECD SPECT healthy brain perfusion analysis using statistical parametric mapping. Biomed Eng-Appl Basis Commun. 2006;18:296-304.

57. Matsuda H, Mizumura S, Soma T, Takemura N. Conversion of brain SPECT images between different collimators and reconstruction processes for analysis using statistical parametric mapping. Nucl Med Commun. 2004;25:67-74. 
58. Matsuda H, Murata M, Mukai $Y$, Sako K, Ono H, Toyama $\mathrm{H}$, et al. Japanese multicenter database of healthy controls for [123 I] FP-CIT SPECT. Eur J Nucl Med Mol Imaging. 2018;45:1405-16.

59. Van Laere K, Koole M, Versijpt J, Vandenberghe S, Brans B, D'Asseler Y, et al. Transfer of normal 99m Tc-ECD brain SPET databases between different gamma cameras. Eur J Nucl Med Mol. 2001;28:435-49.

60. Matsuda H, Mizumura S, Nagao T, Ota T, Iizuka T, Nemoto K, et al. An easy Z-score imaging system for discrimination between very early Alzheimer's disease and controls using brain perfusion SPECT in a multicentre study. Nucl Med Commun. 2007;28:199-205.

61. Stockbridge HL, Lewis D, Eisenberg B, Lee M, Schacher S, van Belle G, et al. Brain SPECT: a controlled, blinded assessment of intra-reader and inter-reader agreement. Nucl Med Commun. 2002;23:537-44.

62. Hellman RS, Tikofsky RS, Heertum Van R, Coade G, Carretta R, Hoffmann RG. A multi-institutional study of interobserver agreement in the evaluation of dementia with $\mathrm{rCBF} /$ SPET technetium-99m exametazime (HMPAO). Eur J Nucl Med. 1994;21:306-13.

63. Pasquier F, Lavenu I, Lebert F, Jacob B, Steinling M, Petit H. The use of SPECT in a multidisciplinary memory clinic. Dement Geriatr Cogn Disord. 1997;8:85-91.

64. Doran M, Vinjamuri S, Collins J, Parker D, Larner AJ. Single-photon emission computed tomography perfusion imaging in the differential diagnosis of dementia: a retrospective regional audit. Int J Clin Pract. 2005;59:496-500.

65. Barnes A, Lusman D, Patterson J, Brown D, Wyper D. The use of Statistical Parametric Mapping (SPM96) as a decision aid in the differential diagnosis of dementia using 99mTc-HMPAO SPECT. Behav Neurol. 2000;12:77-86.

66. Jagust W, Thisted R, Devous MD Sr, Van Heertum R, Mayberg H, Jobst K, et al. SPECT perfusion imaging in the diagnosis of Alzheimer's disease: a clinical- pathologic study. Neurology. 2001;56:950-6.

67. Nitrini R, Buchpiguel CA, Caramelli P, Bahia VS, Mathias SC, Nascimento CM, et al. SPECT in Alzheimer's disease: features associated with bilateral parietotemporal hypoperfusion. Acta Neurol Scand. 2000;101:172-6.

68. Cappa A, Calcagni ML, Villa G, Giordano A, Marra C, De Rossi G, et al. Brain perfusion abnormalities in Alzheimer's disease: comparison between patients with focal temporal lobe dysfunction and patients with diffuse cognitive impairment. J Neurol Neurosurg Psychiatry. 2001;70:22-7.

69. Nieto JCR. Frontotemporal dementia: clinical, neuropsychological, and neuroimaging description. Colomb Med (Cali). 2014;45:122-6.

70. McNeill R, Sare GM, Manoharan M, Testa HJ, Mann DMA, Neary D, et al. Accuracy of SPECT in differentiating frontotemporal dementia from Alzheimer's disease. J Neurol Neurosurg Psychiatry. 2007;78:350-5.

71. Bigler ED, Maxwell WL. Neuropathology of mild traumatic brain injury: relationship to neuroimaging findings. Brain Imaging Behav. 2012;6:108-36.

72. Abu-Judeh HH, Parker R, Aleksic S, Singh ML, Naddaf S, Atay S, et al. SPECT brain perfusion findings in mild or moderate traumatic brain injury. Nucl Med Rev Cent East Eur. 2000;3:5-11. 